\title{
A Dynamical Alternating Direction Multiplier Method for Two-Block Optimization Problems
}

\author{
Miantao Chao · Liqun Liu
}

Received: date / Accepted: date

\begin{abstract}
In this paper, we propose a dynamic alternating direction method of multipliers for two-block separable optimization problems. The well-known classical ADMM can be obtained after the time discretization of the dynamical system. Under suitable condition, we prove that the trajectory asymptotically converges to a saddle point of the Lagrangian function of the problems. When the coefficient matrices in the constrain$\mathrm{t}$ are identiy matrices, we prove the worst-case $O\left(\frac{1}{t}\right)$ convergence rate in ergodic sense.
\end{abstract}

Keywords Dynamical system · Alternating direction multiplier method · Lyapunov function · Convergence analysis

\section{Introduction and preliminaries}

In this paper, we study the following two-block separable optimization problem:

$$
\begin{aligned}
& \min _{x \in \mathcal{H}, z \in \mathcal{G}} f(x)+g(z) \\
& \text { s.t. } \quad A x+B z=b,
\end{aligned}
$$

where $\mathcal{H}, \mathcal{G}$ and $\mathcal{K}$ are real Hilbert spaces, $f: \mathcal{H} \rightarrow$ $\bar{\Re}:=\Re \cup\{+\infty\}$ and $g: \mathcal{G} \rightarrow \bar{\Re}$ are both proper, convex and lower semicontinuous functions, $A$ and $B$ are linear continuous operators such that $A \neq 0$ and $b \in \mathcal{K}$.

\section{Miantao Chao}

College of Mathematics and Information Science, Guangxi

University, Nanning, Guangxi 530004, China

E-mail: chaomiantao@126.com

Liqun Liu

College of Mathematics and Information Science, Guangxi

University, Nanning, Guangxi 530004, China
The Lagrangian function of problem (1.1) is defined:

$\mathcal{L}: \mathcal{H} \times \mathcal{G} \times \mathcal{K} \rightarrow \bar{\Re}$,

$\mathcal{L}(x, z, y)=f(x)+g(z)+\langle y, b-A x-B z\rangle$.

A point $\left(x^{*}, z^{*}, y^{*}\right) \in \mathcal{H} \times \mathcal{G} \times \mathcal{K}$ is called the saddle point of $\mathcal{L}$, if

$\mathcal{L}\left(x^{*}, z^{*}, y\right) \leq \mathcal{L}\left(x^{*}, z^{*}, y^{*}\right) \leq \mathcal{L}\left(x, z, y^{*}\right)$,

$\forall(x, z, y) \in \mathcal{H} \times \mathcal{G} \times \mathcal{K}$.

As we all know, $\left(x^{*}, z^{*}, y^{*}\right)$ is the saddle point of $\mathcal{L}$ if and only if $\left(x^{*}, z^{*}\right)$ is the optimal solution of (1.1) and $y^{*}$ is the optimal solution of its Fenchel-Rockafellar dual problem

$\sup _{y \in \mathcal{K}}\left\{-f^{*}\left(A^{*} y\right)-g^{*}\left(B^{*} y\right)+\langle y, b\rangle\right\}$,

and the optimal objective values of (1.1) and (1.2) are consistent. In the formulation of (1.2),

$$
f^{*}: \mathcal{H} \rightarrow \bar{\Re}, f^{*}(u)=\sup _{x \in \mathcal{H}}\{\langle u, x\rangle-f(x)\}
$$

and

$$
g^{*}: \mathcal{G} \rightarrow \bar{\Re}, g^{*}(v)=\sup _{z \in \mathcal{G}}\{\langle v, z\rangle-g(z)\},
$$

are the conjugate functions of $f$ and $g$, respectively.

The set of saddle points is nonempty if and only if (1.1) has an optimal solution and satisfies the following Attouch-Brezis regularity conditions:

$b \in \operatorname{sqri}(A($ domf $)+B($ domg $))$,

where $\operatorname{sqri} Q:=\left\{x \in Q: \cup_{\lambda>0} \lambda(Q-x)\right.$ is a closed linear subspace of $\left.\mathcal{G}\right\}$

For more information about convex duality theory and regularity conditions, we recommend the readers to refer to references $[9,10,11]$. 
The convex subdifferential of $f$ is defined as

$\partial f(x)=\{u \in \mathcal{H}: f(y) \geq f(x)+\langle u, y-x\rangle, \forall y \in \mathcal{H}\}$,

if $f(x) \in \Re$, and as $\partial f(x)=\varnothing$, otherwise.

In addition, if $f$ is $\sigma_{f}$-strongly convex, then $\partial f$ meet$\mathrm{s}$ the strong monotonicity property:

$\langle u-v, x-y\rangle \geq \sigma_{f}\|x-y\|^{2}, \forall u \in \partial f(x), v \in \partial f(y)$.

The optimality conditions of problems (1.1)-(1.2) are as follows:

$A^{*} y^{*} \in \partial f\left(x^{*}\right), B^{*} y^{*} \in \partial g\left(z^{*}\right)$ and $A x^{*}+B z^{*}=b$.

Remark 1 If $f$ and $g$ are both strong convex functions, $\left(x_{1}^{*}, z_{1}^{*}, y_{1}^{*}\right)$ and $\left(x_{2}^{*}, z_{2}^{*}, y_{2}^{*}\right)$ are two saddle points of the Lagrangian $\mathcal{L}$, then $x_{1}^{*}=x_{2}^{*}$ and $z_{1}^{*}=z_{2}^{*}$. This follows easily from (1.4), by using the strong monotonicity of $\partial f$ and $\partial g$.

As is known to all, if problem (1.1) meets the restriction conditions (1.3) and has an optimal solution $\left(x^{*}, z^{*}\right)$, then we have the optimal solution $y^{*}$ of problem (1.2) that makes equation (1.4) valid. On the contrary, $\left(x^{*}, z^{*}, y^{*}\right)$ satisfies the optimality condition (1.4) if and only if $\left(x^{*}, z^{*}\right)$ is the optimal solution of (1.1) and $y^{*}$ is the optimal solution of (1.2).

Let $S_{+}(\mathcal{H})$ denote the set of continuous, linear, positive semidefinite and self-adjoint operators from $\mathcal{H}$ to $\mathcal{H}$. For $M \in S_{+}(\mathcal{H})$ we define the seminorm $\|\cdot\|_{M}$ : $\mathcal{H} \rightarrow \Re$ with $\|x\|_{M}=\sqrt{\langle x, M x\rangle}$. We recall the following Loewner partial ordering on $S_{+}(\mathcal{H})$ : for $M_{1}, M_{2} \in$ $S_{+}(\mathcal{H})$,

$M_{1} \succeq M_{2} \Leftrightarrow\|x\|_{M_{1}} \geq\|x\|_{M_{2}}, \forall x \in \mathcal{H}$.

The dynamical system proposed and studied in this paper is as follows:

$$
\left\{\begin{aligned}
& \dot{x}(t)+x(t) \in\left(\partial f+c(t) A^{*} A\right)^{-1}\left(A^{*} y(t)\right. \\
&\left.-c(t) A^{*} B z(t)+c(t) A^{*} b\right) \\
& \dot{z}(t)+z(t) \in\left(\partial g+c(t) B^{*} B\right)^{-1}\left(B^{*} y(t)\right. \\
&\left.-c(t) B^{*} A(\dot{x}(t)+x(t))+c(t) B^{*} b\right) \\
& \dot{y}(t)= \sigma c(t)(b-A(\dot{x}(t)+x(t))-B(\dot{z}(t)+z(t))) \\
& x(0)=x^{0} \in \mathcal{H}, z(0)=z^{0} \in \mathcal{G}, y(0)=y^{0} \in \mathcal{K}
\end{aligned}\right.
$$

where $\sigma>0, c(t)>0$ for all $t \in[0,+\infty)$.

In fact, since the $1970 \mathrm{~s}$, dynamical systems related to the problem of approximate monotone inclusion and optimization have attracted extensive attention (see Brezis Baillon and Bruck, Crandall and Pazy [8, 14, 15, 16]). This is not only because of their inherent importance in such fields as differential equations and applied functional analysis, but also because they are considered a useful tool for finding and studying numerical algorithms for optimization problems resulting from discretization of continuous dynamical systems. In the field of optimization, the dynamic method of iterative method can provide an in-depth understanding of the expected behavior of the method. In addition, the methods and techniques we used in the continuous case can be applied to the results of the discretization algorithm. For more information on the relationship between continuous and discrete dynamics, the reader can refer to the reference [1].

In recent years, there are some researches on the dynamical system of numerical algorithm. In [5], Abbas and Attouch considered a forward backward dynamical system. Banert and Bot in [7] introduced a forward-backward-forward dynamical system. Bot and others proposed in [13] an implicit dynamical system. Csetnek and others considered a Douglas-Rachford type dynamical system in [17]. And recently, a primal-dual dynamical system was introduced in [2], and a proximal alternating minimization algorithm dynamical system was proposed in [3].

One of the motivations for our study is the classical ADMM algorithm resulting from the discretization of this dynamical system, which we will see in the Remark 3. The algorithm and its variants are widely used to solve various optimization problems, and it is one of the research hotspots in the optimization field. Therefore, it is of great significance for us to further understand the algorithm by using the continuous correspondence of its algorithm through tools such as differential equations. Here, we compare the differences between this paper and the existing literatures on the discrete algorithms corresponding to the dynamical system. In reference [2], the discrete algorithm for dynamical systems is a combination of the linearized proximal method of multipliers and the proximal ADMM algorithm. In reference [3], the discrete algorithm corresponding to the dynamical system is the proximal AMA algorithm. In reference [4], the discrete algorithms corresponding to the dynamical system are some fast inertial approximate ADMM algorithms.

In addition, in the process of solving the problem, this paper uses tools similar to literature [2] and [3]. Next, we focus on the differences between this paper and these two literatures. First of all, the structure of the problem we consider is different. Compared with [2], our constraint set contains two linear operators. Secondly, our dynamical system corresponds to different discrete algorithms, which have been mentioned above. Finally, in our dynamical system, there is an additional 
parameter $c$ (it is a time variable), which is differen$\mathrm{t}$ from reference [2], and we also have another additional parameter $\sigma$, which is different from reference [3] (the motivation of taking variables $c$ and $\sigma$ comes from reference [6], where the numerical scheme ADMM also contains variable parameters $c$ and $\sigma$ ).

The follow-up organization of the article is as follows. In the next section, we obtain the classical ADMM algorithm by explicit time discretization of the dynamical system. In addition, we give the concept of solution related to the dynamical system (1.5) and the equivalent expression of (1.5). Furthermore, we give a numerical example to illustrate how the selection of parameters affects the convergence of the trajectory. In the third section, we focus on proving the existence and uniqueness of strong global solutions of the dynamical systems (1.5). The analysis mainly depends on CauchyLipschitz-Picard theorem. In the section 4, relying on a continuous variant of the Opial lemma and a Lyapunov analysis, where the selection of appropriate energy functions plays a key role, we prove that the solution trajectory of the proposed dynamical system converges weakly to the saddle point of Lagrange $\mathcal{L}$. Furthermore, when the coefficient matrices in the constraint are identiy matrices, we prove the worst-case $O\left(\frac{1}{t}\right)$ convergence rate in ergodic sense. In addition, we demonstrate how the dynamical alternating direction method of multipliers can be applied in the absence of a strongly convex block.

\section{Solution concept, discretization, example}

Let's review the definition of a locally absolutely continuous map before we specify what do we mean by a solution of (1.5).

Definition 1 A function $x:[0, \infty) \rightarrow \mathcal{H}$ is said to be locally absolutely continuous, if it is absolutely continuous on every interval $[0, T], T>0$; that is, for every $T>0$ there exists an integrable function $y:[0, T] \rightarrow \mathcal{H}$ such that

$$
x(t)=x(0)+\int_{0}^{t} y(s) d s \quad \forall t \in[0, T] .
$$

Remark 2 (a)Every absolutely continuous function is differentiable almost everywhere.

(b)Let $T>0$, and $x:[0, T] \rightarrow \mathcal{H}$ is an absolutely continuous function. This is equivalent to: for every $\epsilon>$ 0 there exists $\eta>0$ such that for any finite family of intervals $I_{k}=\left(a_{k}, b_{k}\right) \subseteq[0, T]$ the following property holds: for any subfamily of disjoint intervals $I_{j}$ with $\sum_{j}\left|b_{j}-a_{j}\right|<\eta$ it holds

$$
\sum_{j}\left\|x\left(b_{j}\right)-x\left(a_{j}\right)\right\|<\epsilon .
$$

From this characterization it is easy to see that, if $M: \mathcal{H} \rightarrow \mathcal{H}$ is $\mathcal{L}$-Lipschitz continuous with $\mathcal{L} \geq 0$, then the function $z=M \circ x$ is absolutely continuous, too. This means that $z$ is differentiable almost everywhere and $\|\dot{z}(\cdot)\| \leq \mathcal{L}\|\dot{x}(\cdot)\|$ holds almost everywhere.

The following definition specifies which type of solutions we consider in the analysis of the dynamical $\operatorname{system}(1.5)$.

Definition 2 Let $\left(x^{0}, z^{0}, y^{0}\right) \in \mathcal{H} \times \mathcal{G} \times \mathcal{K}, \sigma>0$. We say that the function $(x, z, y):[0,+\infty) \rightarrow \mathcal{H} \times \mathcal{G} \times$ $\mathcal{K}$ is a strong global solutions of (1.5), if the following properties are satisfied:

(i) the functions $x, z, y$ are locally absolutely continuous;

(ii) for almost every $t \in[0,+\infty)$

$$
\begin{aligned}
& \dot{x}(t)+x(t) \in\left(\partial f+c(t) A^{*} A\right)^{-1}\left(A^{*} y(t)-c(t) A^{*} B z(t)\right. \\
&\left.+c(t) A^{*} b\right) \\
& \dot{z}(t)+z(t) \in\left(\partial g+c(t) B^{*} B\right)^{-1}\left(B^{*} y(t)-c(t) B^{*} A(\dot{x}(t)\right. \\
&\left.+x(t))+c(t) B^{*} b\right) \\
& \dot{y}(t)=\sigma c(t)(b-A(\dot{x}(t)+x(t))-B(\dot{z}(t)+z(t))) \\
& \text { (iii) } x(0)=x^{0}, z(0)=z^{0}, \text { and } y(0)=y^{0} .
\end{aligned}
$$

Remark 3 Let us consider a discretization of the considered dynamical system. The first two inclusions in (1.5) can be written in an equivalent way as

$$
\begin{aligned}
0 \in & \partial f(\dot{x}(t)+x(t))-A^{*} y(t)+c(t) A^{*} A(\dot{x}(t)+x(t)) \\
& +c(t) A^{*} B z(t)-c(t) A^{*} b
\end{aligned}
$$

$$
\begin{gathered}
0 \in \partial g(\dot{z}(t)+z(t))-B^{*} y(t)+c(t) B^{*} A(\dot{x}(t)+x(t)) \\
+c(t) B^{*} B(\dot{z}(t)+z(t))-c(t) B^{*} b
\end{gathered}
$$

for $t \in[0,+\infty)$. Through explicit discretization with respect to the time variable $t$ and constant step size $h_{k} \equiv 1$ (i.e. $x(t) \approx x^{k}$ and $\dot{x}(t) \approx x^{k+1}-x^{k}$ ) we obtain for all $k \geq 0$ the inclusions:

$$
\begin{aligned}
& 0 \in \partial f\left(x^{k+1}\right)-A^{*} y^{k}+c_{k} A^{*} A x^{k+1}+c_{k} A^{*} B z^{k}-c_{k} A^{*} b, \\
& 0 \in \partial g\left(z^{k+1}\right)-B^{*} y^{k}+c_{k} B^{*} A x^{k+1}+c_{k} B^{*} B z^{k+1}-c_{k} B^{*} b .
\end{aligned}
$$

Furthermore, using convex subdifferential calculus this can be written equivalently for all $k \geq 0$ as

$0 \in \partial\left(f(\cdot)-\left\langle y^{k}, A \cdot\right\rangle+\frac{1}{2} c_{k}\left\|A \cdot+B z^{k}-b\right\|^{2}\right)\left(x^{k+1}\right)$, $0 \in \partial\left(g(\cdot)-\left\langle y^{k}, B \cdot\right\rangle+\frac{1}{2} c_{k}\left\|A x^{k+1}+B \cdot-b\right\|^{2}\right)\left(z^{k+1}\right)$.

Hence the dynamical system (1.5) provides through explicit time discretization the following numerical algorithm: 
Choose $\left(x^{0}, z^{0}, y^{0}\right) \in \mathcal{H} \times \mathcal{G} \times \mathcal{K}, \sigma>0$ and $\left(c_{k}\right)_{k \geq 0}$. For all $k \geq 0$ generate the sequence $\left(x^{k}, z^{k}, y^{k}\right)_{k \geq 0}$ as follows:

$$
\left\{\begin{array}{l}
x^{k+1}=\underset{x \in \mathcal{H}}{\operatorname{argmin}}\left\{f(x)-\left\langle y^{k}, A x\right\rangle+\frac{1}{2} c_{k}\left\|A x+B z^{k}-b\right\|^{2}\right\} \\
z^{k+1}=\underset{z \in \mathcal{G}}{\operatorname{argmin}}\left\{g(z)-\left\langle y^{k}, B z\right\rangle+\frac{1}{2} c_{k}\left\|A x^{k+1}+B z-b\right\|^{2}\right\} \\
y^{k+1}=y^{k}+\sigma c_{k}\left(b-A x^{k+1}-B z^{k+1}\right) .
\end{array}\right.
$$

The algorithm above is the classical ADMM algorithm introduced by Glowinski in [6].

Remark 4 In this paper we will often use the following equivalent formulation of the dynamical system(1.5). For $U(t)=(x(t), z(t), y(t)),(1.5)$ can be written as

$\left\{\begin{array}{c}\dot{U}(t)=\Gamma(t, U(t)) \\ U(0)=\left(x^{0}, z^{0}, y^{0}\right),\end{array}\right.$

where

$\Gamma:[0,+\infty) \times \mathcal{H} \times \mathcal{G} \times \mathcal{K} \rightarrow \mathcal{H} \times \mathcal{G} \times \mathcal{K}, \Gamma(t, x, z, y)=(u, v, w)$
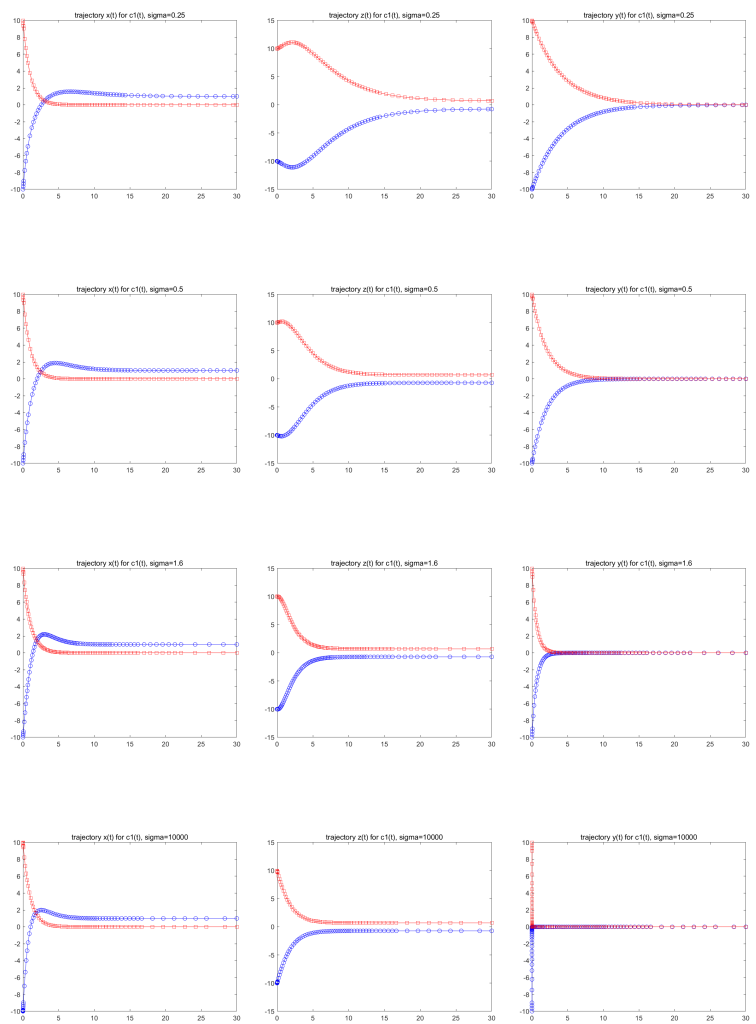

Figure1: First and second column: the primal trajectories $x(t)$ and $z(t)$ converge to the primal optimal solution $(0.9563,-0.2732)$ and $(0.9660,0)$ respectively for $c_{1}(t)=\frac{1}{\sqrt{t+1.1}}+0.01$ and starting point $(-10,10)$. Third column: the dual trajectory $y(t)$ converges to the dual optimal solution $(-0.4173,-0.3555)$ for $c_{1}(t)=\frac{1}{\sqrt{t+1.1}}+0.01$ and starting point $(-10,10)$. is defined as

$$
\begin{aligned}
& u=u(t, x, z, y) \in \underset{p \in \mathcal{H}}{\operatorname{argmin}}\left\{F(t, p)+\frac{c(t)}{2} \| p-\right. \\
& \left.\left(\frac{1}{c(t)} A^{*} y-A^{*}(B z-b)\right) \|^{2}\right\}-x \\
& v=v(t, x, z, y) \in \underset{q \in \mathcal{G}}{\operatorname{argmin}}\left\{G(t, q)+\frac{c(t)}{2} \| q-\right. \\
& \left.\left(\frac{1}{c(t)} B^{*} y-B^{*}(A(x+u)-b)\right) \|^{2}\right\}-z \\
& w=w(t, x, z, y)=\sigma c(t)(b-A(x+u)-B(z+v))
\end{aligned}
$$

with

$F:[0,+\infty) \times \mathcal{H} \rightarrow \bar{\Re}, F(t, p)=f(p)+\frac{c(t)}{2}\left(\|A p\|^{2}-\|p\|^{2}\right)$

and

$G:[0,+\infty) \times \mathcal{G} \rightarrow \bar{\Re}, G(t, q)=g(q)+\frac{c(t)}{2}\left(\|B q\|^{2}-\|q\|^{2}\right)$.

Let $t \in[0,+\infty)$ be fixed. The functions $F(t, \cdot)+$ $\frac{c(t)}{2}\|\cdot-u\|^{2}$ and $G(t, \cdot)+\frac{c(t)}{2}\|\cdot-v\|^{2}$ are proper, convex and lower semicontinuous. If $f(p)$ and $g(q)$ are strong convex, then we can use the sign equal in the first and second relation of $(2.3)$.
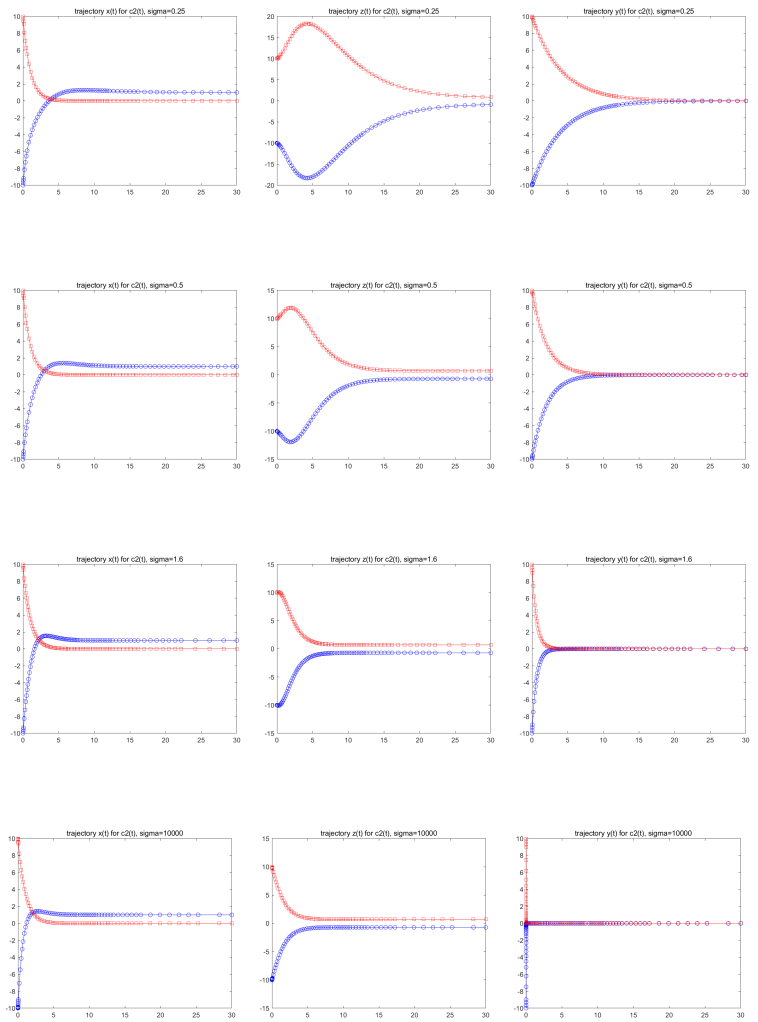

Figure2: First and second column: the primal trajectories $x(t)$ and $z(t)$ converge to the primal optimal solution $(0.9563,-0.2732)$ and $(0.9660,0)$ respectively for $c_{2}(t)=\frac{1}{t+1.1}+0.01$ and starting point $(-10,10)$. Third column: the dual trajectory $y(t)$ converges to the dual optimal solution $(-0.4173,-0.3555)$ for $c_{2}(t)=\frac{1}{t+1.1}+0.01$ and starting point $(-10,10)$. 
Example 1 We consider the following optimization problem

$$
\begin{array}{r}
\inf _{x \in \Re^{2}, z \in \Re^{2}} \frac{1}{2}\|x-d\|^{2}+\frac{1}{2}\|z-d\|^{2} \\
\text { s.t. } \quad A x+B z=0
\end{array}
$$

with

$A=\frac{1}{\sqrt{8}}\left(\begin{array}{cc}2 & 1 \\ -2 & 1\end{array}\right), B=\frac{1}{5}\left(\begin{array}{rr}-3 & 0 \\ 4 & 0\end{array}\right)$ and $d=\left(\begin{array}{l}1 \\ 0\end{array}\right)$,

which is problem (1.1) with $\mathcal{H}=\mathcal{G}=\Re^{2}, f, g$ : $\Re^{2} \rightarrow \Re, f(x)=\frac{1}{2}\|x-d\|^{2}, g(z)=\frac{1}{2}\|z-d\|^{2}$, for every $x \in \Re^{2}$ and $z \in \Re^{2}$. We can confirm that (2.4) has a unique solution, which is $x^{*}=(0.9563,-0.2732)$ and $z^{*}=(0.9660,0)$. The Fenchek-Rockafellar dual problem of $(2.4)$ is

$\sup _{y \in \Re^{2}}\left\{-f^{*}\left(A^{*} y\right)-g^{*}\left(B^{*} y\right)\right\}$
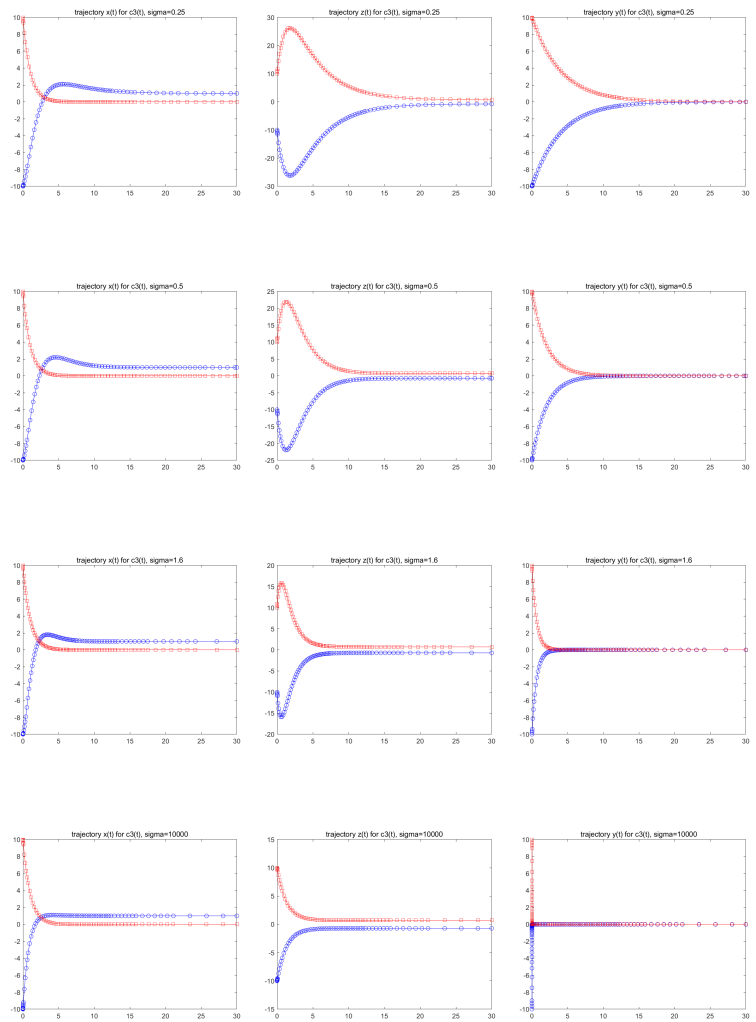

Figure3: First and second column: the primal trajectories $x(t)$ and $z(t)$ converge to the primal optimal solution $(0.9563,-0.2732)$ and $(0.9660,0)$ respectively for constant $c_{3}(t)=0.25$ and starting point $(-10,10)$. Third column: the dual trajectory $y(t)$ converges to the dual optimal solution $(-0.4173,-0.3555)$ for $c_{3}(t)=0.25$ and starting point $(-10,10)$. which is equivalent to

$-\inf _{y \in \Re^{2}}\left\{f^{*}\left(A^{*} y\right)+g^{*}\left(B^{*} y\right)\right\}$.

And

$\sup _{y \in \Re^{2}}\left\{\frac{1}{2}\left\|A^{*} y\right\|^{2}+\frac{1}{2}\left\|B^{*} y\right\|^{2}-\left\langle\left(A^{*}+B^{*}\right) y, d\right\rangle\right\}$,

where the unique optimal solution is $y^{*}=(-0.4173$,$0.3555)$.

For $U(t)=(x(t), z(t), y(t))$, we can write the dynamical system for this problem (1.5) similarly as in Remark 4

$\left\{\begin{array}{c}\dot{U}(t)=\Gamma(t, U(t)) \\ U(0)=\left(x^{0}, z^{0}, y^{0}\right),\end{array}\right.$

where

$\Gamma:[0,+\infty) \times \mathcal{H} \times \mathcal{G} \times \mathcal{K} \rightarrow \mathcal{H} \times \mathcal{G} \times \mathcal{K}, \Gamma\left(t, u_{1}, u_{2}, u_{3}\right)=\left(u_{4}, u_{5}, u_{6}\right)$,
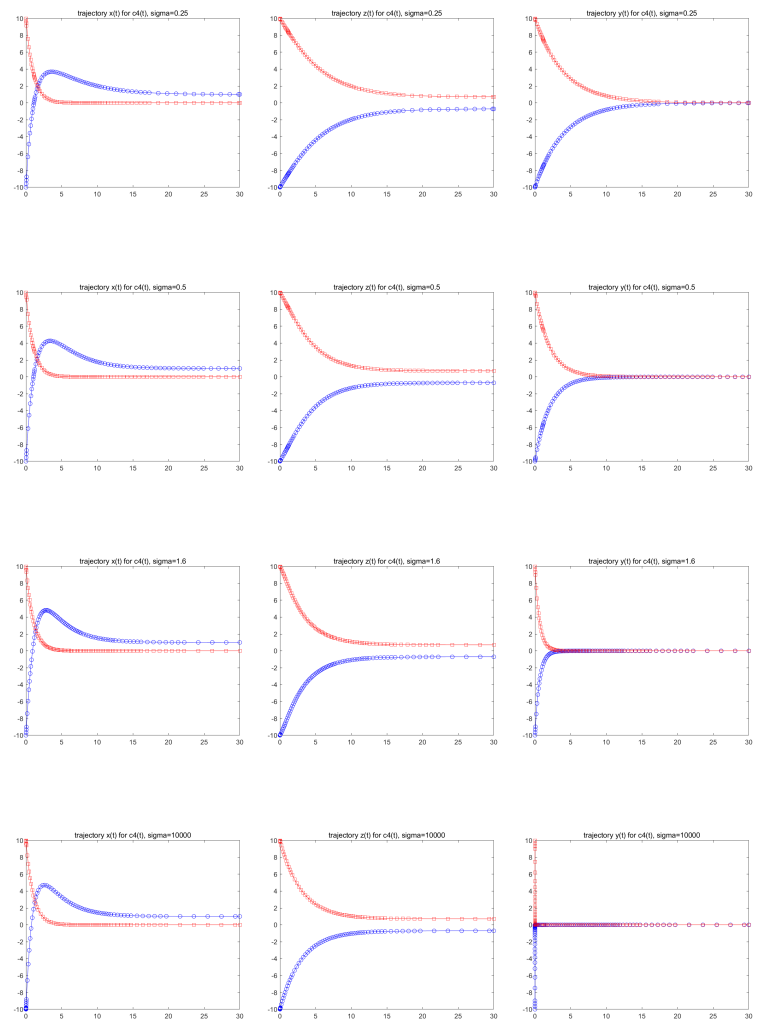

Figure4: First and second column: the primal trajectories $x(t)$ and $z(t)$ converge to the primal optimal solution $(0.9563,-0.2732)$ and $(0.9660,0)$ respectively for constant $c_{4}(t)=1.99$ and starting point $(-10,10)$. Third column: the dual trajectory $y(t)$ converges to the dual optimal solution $(-0.4173,-0.3555)$ for $c_{4}(t)=1.99$ and starting point $(-10,10)$. 
is defined as

$$
\left\{\begin{aligned}
u_{4}= & \underset{p \in \mathcal{H}}{\operatorname{argmin}}\left\{f(p)+\frac{c(t)}{2}\left(\|A p\|^{2}-\|p\|^{2}\right)+\frac{c(t)}{2} \| p-\right. \\
& \left.\left(\frac{1}{c(t)} A^{*} y-A^{*}(B z-b)\right) \|^{2}\right\}-u_{1} \\
= & \left(c(t) A^{*} A+I d\right)^{-1}\left(A^{*} u_{3}-c(t) A^{*} B u_{2}+d\right)-u_{1} \\
u_{5}= & \underset{q \in \mathcal{G}}{\operatorname{argmin}}\left\{g(q)+\frac{c(t)}{2}\left(\|B q\|^{2}-\|q\|^{2}\right)+\frac{c(t)}{2} \| q-\right. \\
& \left.\left(\frac{1}{c(t)} B^{*} y-B^{*}(A(x+u)-b)\right) \|^{2}\right\}-u_{2} \\
= & \left(c(t) B^{*} B+I d\right)^{-1}\left(B^{*} u_{3}-c(t) B^{*} A\left(u_{1}+u(4)\right)+d\right) \\
& -u_{2} \\
u_{6}= & \sigma c(t)\left(-A\left(u_{1}+u_{4}\right)-B\left(u_{2}+u_{5}\right)\right) .
\end{aligned}\right.
$$

We solved the dynamical system (1.5) with the starting points $x^{0}=(-10,10), z^{0}=(-10,10)$ and $y^{0}=$ $(-10,10)$ in the case when $c(t)>0$ for every $t \in$ $[0,+\infty)$.

According to Theorem 2, it has to be satisfied for an $\epsilon>0$ that $0<c(t)<\min \left\{\frac{\sigma_{f}}{\|A\|^{2}}, \frac{\sigma_{g}}{\|B\|^{2}}\right\}-\epsilon$ and $\dot{c}(t) \leq 0$ for all $t$ in order to ensure asymptotic convergence of the trajectory, where $\sigma_{f}, \sigma_{g}$ are the strong convexity parameter of $f(x)$ and $g(z)$ respectively (here $\sigma_{f}=\sigma_{g}=1$ ), and that $c(t)$ is Lipschitz continuous. For $c(t)=c$ constant, we can choose $c$ such that $c<\min \left\{\frac{2 \sigma_{f}}{\|A\|^{2}}, \frac{2 \sigma_{g}}{\|B\|^{2}}\right\}-\epsilon$. Since $\|A\|^{2}=1$ and $\|B\|^{2}=1$, we choose $c_{1}(t)=\frac{1}{\sqrt{t+1.1}}+0.01$, $c_{2}(t)=\frac{1}{t+1.1}+0.01, c_{3}(t)=0.25$, and $c_{4}(t)=1.99$ to satisfy the above conditions. From Figure 1-4 above, we can see that the larger $\sigma$ is, the faster all three trajectories converge, which is independent of the choice of $c(t)$. Furthermore, we can also find that when the $\sigma$ value is certain large, the growth rate of the primal trajectories $x(t)$ and $z(t)$ are not obvious as $\sigma$ continues to increase.

\section{Existence and uniqueness of the trajectories}

In this section we will prove the existence and uniqueness of the trajectories generated by the dynamical system (1.5). Let's start with two very important lemmas, which are often used in subsequent proofs. To this end, we make the following assumption

$(\mathcal{P}) \quad f$ and $g$ are $\sigma_{f}-$ and $\sigma_{g}-$ strongly convex functions, respectively.

Lemma 1 Let assumption $(\mathcal{P})$ hold true and $t \in[0,+\infty)$. Then the operator

$$
K_{t}: \mathcal{H} \rightarrow \mathcal{H}, K_{t}(u)=\underset{x \in \mathcal{H}}{\operatorname{argmin}}\left(F(t, x)+\frac{c(t)}{2}\|x-u\|^{2}\right)
$$

is $\frac{c(t)}{\sigma_{f}}$-Lipschitz continuous and the operator

$$
J_{t}: \mathcal{G} \rightarrow \mathcal{G}, J_{t}(v)=\underset{q \in \mathcal{G}}{\operatorname{argmin}}\left(G(t, z)+\frac{c(t)}{2}\|z-v\|^{2}\right)
$$

is $\frac{c(t)}{\sigma_{g}}$-Lipschitz continuous.

Proof See Appendix A.

Lemma 2 Let assumption $(\mathcal{P})$ hold true, $(x, z, y) \in$ $\mathcal{H} \times \mathcal{G} \times \mathcal{K}$ and the maps

$$
\begin{aligned}
R_{(x, z, y)}(t)=\underset{u \in \mathcal{H}}{\operatorname{argmin}}\left\{F(t, u)+\frac{c(t)}{2} \| u-\left(\frac{1}{c(t)} A^{*} y-\right.\right. \\
\left.\left.A^{*} B z+A^{*} b\right) \|^{2}\right\}-x, \\
Q_{(x, z, y)}(t)=\underset{v \in \mathcal{G}}{\operatorname{argmin}}\left\{G(t, v)+\frac{c(t)}{2} \| v-\left(\frac{1}{c(t)} B^{*} y-\right.\right. \\
\left.\left.B^{*} A\left(R_{(x, z, y)}(t)+x\right)+B^{*} b\right) \|^{2}\right\}-z,
\end{aligned}
$$

and

$$
\begin{aligned}
P_{(x, z, y)}(t)= & \sigma c(t)\left(b-A\left(R_{(x, z, y)}(t)+x\right)-B\left(Q_{(x, z, y)}(t)\right.\right. \\
& +z)) .
\end{aligned}
$$

Then the following holds for every $t, r \in[0, \infty)$.

(i) $\left\|R_{(x, z, y)}(t)-R_{(x, z, y)}(r)\right\|$

$$
\begin{aligned}
\leq & \left(\frac{\|A\|\|B\|\left\|Q_{(x, z, y)}(r)\right\|}{\sigma_{f}}\right. \\
& \left.+\frac{\|A\|\left\|P_{(x, z, y)}(r)\right\|}{\sigma \sigma_{f} c(r)}\right)|c(t)-c(r)| .
\end{aligned}
$$

(ii) $\left\|Q_{(x, z, y)}(t)-Q_{(x, z, y)}(r)\right\|$

$$
\begin{aligned}
\leq & \left(\frac{c(t)\|A\|^{2}\|B\|^{2}\left\|Q_{(x, z, y)}(r)\right\|}{\sigma_{f} \sigma_{g}}\right. \\
& \left.+\frac{\left(c(t)\|A\|^{2}+\sigma_{f}\right)\|B\|\left\|P_{(x, z, y)}(r)\right\|}{\sigma \sigma_{f} \sigma_{g} c(r)}\right)|c(t)-c(r)| .
\end{aligned}
$$

Proof See Appendix A.

With these estimates, we can now prove the existence and uniqueness of trajectories.

Theorem 1 Assume that $(\mathcal{P})$ holds and $0<\inf _{t \geq 0} c(t) \leq$ $\sup _{t>0} c(t)<+\infty$. Then, for every starting points $\left(x^{0}, z^{0}, y^{0}\right)$ $\rightarrow \mathcal{H} \times \mathcal{G} \times \mathcal{K}$, the dynamical system (1.5) has a unique strong global solution $(x, z, y):[0,+\infty) \rightarrow \mathcal{H} \times \mathcal{G} \times \mathcal{K}$.

Proof See Appendix A. 


\section{Convergence of the trajectories}

4.1 The dynamical alternating direction multiplier method for problem (1.1) with stongly convex function

In this section, we will first give some results, which we will use them to prove the convergence of the trajectories of the dynamical system (1.5). In the following the real vector space

$$
\mathcal{L}(\mathcal{H}):=\{A: \mathcal{H} \rightarrow \mathcal{H}: A \text { is linear and continuous }\}
$$

is endowed with the norm $\|A\|=\sup _{\|x\| \leq 1}\|A x\|$.

Definition 3 The map $M:[0,+\infty) \rightarrow \mathcal{L}(\mathcal{H})$ is said to be derivable at $t_{0} \in[0,+\infty)$, if the limit

$\lim _{h \rightarrow 0} \frac{M\left(t_{0}+h\right)-M\left(t_{0}\right)}{h}$

taken with respect to the norm topology of $\mathcal{L}(\mathcal{H})$ exists. When this is the case, we denote by $\dot{M}\left(t_{0}\right) \in \mathcal{L}(\mathcal{H})$ the value of the limit.

In case $M:[0,+\infty) \rightarrow \mathcal{L}(\mathcal{H})$ is derivable at $t_{0} \in$ $[0,+\infty)$ and $x, y:[0,+\infty) \rightarrow \mathcal{H}$ are also derivable at $t_{0}$, we will use the following formula:

$$
\begin{aligned}
& \left.\frac{d}{d t}\langle M(t) x(t), y(t)\rangle\right|_{t=t_{0}} \\
& =\left\langle\dot{M}\left(t_{0}\right) x\left(t_{0}\right), y\left(t_{0}\right)\right\rangle+\left\langle M\left(t_{0}\right) \dot{x}\left(t_{0}\right), y\left(t_{0}\right)\right\rangle \\
& \quad+\left\langle M\left(t_{0}\right) x\left(t_{0}\right), \dot{y}\left(t_{0}\right)\right\rangle .
\end{aligned}
$$

We start with a result where we show that under appropriate conditions the second derivatives of the trajectories exist almost everywhere and give also an upper bound on their norms. This will be used in the proof of the main result Theorem 2 .

Lemma 3 Assume that $(\mathcal{P})$ holds, $c$ is locally absolutely continuous and $0<\inf _{t \geq 0} c(t) \leq \sup _{t>0} c(t)<+\infty$. For a given starting point $\left(x^{0}, z^{0}, y^{0}\right) \in \mathcal{H} \times \mathcal{G} \times \mathcal{K}$, let $(x, z, y):[0,+\infty) \rightarrow \mathcal{H} \times \mathcal{G} \times \mathcal{K}$ be the unique strong global solution of the dynamical system (1.5). Then

$$
t \rightarrow(\dot{x}(t), \dot{z}(t), \dot{y}(t))
$$

is locally absolutely continuous, hence $(\ddot{x}(t), \ddot{z}(t), \ddot{y}(t))$ exists for almost every $t \in[0,+\infty)$ and there exists $L>0$ such that

$$
\begin{aligned}
& \|\ddot{x}(t)\|+\|\ddot{z}(t)\| \\
& \leq L(\|\dot{x}(t)\|+\|\dot{z}(t)\|+\|\dot{y}(t)\|+(\|\dot{z}(t)\|+\|\dot{y}(t)\|)|\dot{c}(t)|)
\end{aligned}
$$

for every $t \in[0,+\infty)$.

Proof See Appendix B.
In the following we recall two results which we need for the asymptotic analysis (see [21]).

Lemma 4 Assume that $u:[0,+\infty) \rightarrow \Re$ is locally absolutely continuous and bounder from below and that there exists $v \in L^{1}([0,+\infty), \Re)$ with the property that for almost every $t \in[0,+\infty)$

$$
\frac{d}{d t} u(t) \leq v(t)
$$

Then there exists $\lim _{t \rightarrow+\infty} u(t) \in \Re$.

Lemma 5 Assume that $1 \leq p<\infty, 1 \leq r \leq \infty, u$ : $[0,+\infty) \rightarrow[0,+\infty)$ is locally absolutely continuous, $u \in$ $L^{p}([0,+\infty), \Re), v:[0,+\infty) \rightarrow \Re, v \in L^{r}([0,+\infty), \Re)$ and for almost every $t \in[0,+\infty)$

$$
\frac{d}{d t} u(t) \leq v(t)
$$

Then there exists $\lim _{t \rightarrow+\infty} u(t)=0$.

By Lyapunov analysis, we obtain the result that the trajectory generated by the dynamic system (1.5) converges to the Lagrange saddle point of problem (1.1).

Theorem 2 In the setting of the optimization problem (1.1), assume that $(\mathcal{P})$ holds, the set of saddle points of the Lagrangian $\mathcal{L}$ is nonempty, for $0<\epsilon<\frac{1}{2} \min \left\{\frac{\sigma_{f}}{\|A\|^{2}}, \frac{\sigma_{g}}{\|B\|^{2}}\right\}$ the function $c:[0,+\infty) \rightarrow\left[\epsilon, \min \left\{\frac{\sigma_{f}}{\|A\|^{2}}, \frac{\sigma_{g}}{\|B\|^{2}}\right\}-\epsilon\right]$ is Lipschitz continuous and monotonically decreasing. If $c(t)$ is a constant function, namely $c(t)=c$ for all $t \in[0,+\infty)$, then it is enough to assume that $\epsilon \leq c \leq \min \left\{\frac{2 \sigma_{f}}{\|A\|^{2}}, \frac{2 \sigma_{g}}{\|B\|^{2}}\right\}-\epsilon$. For an arbitrary starting point $\left(x^{0}, z^{0}, y^{0}\right) \in \mathcal{H} \times \mathcal{G} \times \mathcal{K}$, let $(x, z, y):[0,+\infty) \rightarrow \mathcal{H} \times \mathcal{G} \times \mathcal{K}$ be the unique strong global solution of the dynamical system (1.5). Then the trajectory $(x(t), z(t), y(t))$ converges weakly to a saddle point of $\mathcal{L}$ as $t \rightarrow \infty$.

Proof See Appendix B.

In the case of $A=B=I d$, we provide rates for both the violation of the feasibility condition by the ergodic trajectories and the convergence of the objective function along these ergodic trajectories to its minimal value.

Theorem 3 Let $0<m \leq 4 \sigma$. In the setting of the optimization problem (1.1), for the case of $A=B=I d$, assume that $(\mathcal{P})$ holds, the set of saddle points of the Lagrangian $\mathcal{L}$ is nonempty, $c(t)=c>0$ for all $t \in$ $[0,+\infty)$. For an arbitrary starting point $\left(x^{0}, z^{0}, y^{0}\right) \in$ $\mathcal{H} \times \mathcal{G} \times \mathcal{K}$, let $(x, z, y):[0,+\infty) \rightarrow \mathcal{H} \times \mathcal{G} \times \mathcal{K}$ be the unique strong global solution of the dynamical system (1.5). Consider further for every $t \in(0,+\infty)$ the ergodic trajectories 


$$
\tilde{x}(t)=\frac{1}{t} \int_{0}^{t}(\dot{x}(s)+x(s)) d s
$$

and

$$
\tilde{z}(t)=\frac{1}{t} \int_{0}^{t}(\dot{z}(s)+z(s)) d s .
$$

Then there exists $K \geq 0$ such that for every $t \in(0,+\infty)$

$$
\|b-\tilde{x}-\tilde{z}\| \leq \frac{K}{t}
$$

In addition, for every $\bar{x} \in \mathcal{H}, \bar{z} \in \mathcal{G}, \bar{x}+\bar{z}=b$ and for every $t \in(0,+\infty)$ such that $(\tilde{x}(t), \tilde{z}(t)) \in \operatorname{dom} f \times \operatorname{domg}$, one has

$$
\begin{aligned}
& (f(\tilde{x}(t))+g(\tilde{z}(t)))-(f(\bar{x})+g(\bar{z})) \\
& \leq \frac{\left\|\left(x^{0}, z^{0}, y^{0}\right)-(\bar{x}, \bar{z}, 0)\right\|_{W(0)}^{2}}{2 t} .
\end{aligned}
$$

Proof See Appendix B.

4.2 The dynamical alternating direction multiplier method for problem (1.1) in the absence of a stongly convex block

In the section 4.1, We assume that both $f$ and $g$ are strongly convex functions. However, in many applications, there is only one block in the objective function is strongly convex. In this section, we demonstrate how to properly apply the dynamical alternating direction method of multipliers to minimize the convex optimization problem in a Hilbert space when there is only one strong convex block in the objective function. We considered the following convex programming questions:

$$
\begin{gathered}
\min _{x \in \mathcal{H}, z \in \mathcal{G}} h(x)+\theta(z) \\
\text { s.t. } \quad A x+z=b,
\end{gathered}
$$

where $\mathcal{H}, \mathcal{G}$ and $\mathcal{K}$ are real Hilbert spaces, $h: \mathcal{H} \rightarrow$ $\bar{\Re}:=\Re \cup\{ \pm \infty\}$ is a proper, $\sigma_{h}$-strongly convex and lower semicontinuous function, $g: \mathcal{G} \rightarrow \bar{\Re}:=\Re \cup\{ \pm \infty\}$ is a proper, convex and lower semicontinuous function, $A$ is a nonzero linear continuous operators and $b \in \mathcal{K}$.

In (4.3), $\theta$ is not a strongly convex function, so we cannot directly apply the dynamical alternating direction method of multipliers to obtain the similar convergence results in section 4.1. In order to obtain strongly convex terms, we restate the problem as the following equivalent problem:

$$
\begin{array}{r}
\min _{x \in \mathcal{H}, z \in \mathcal{G}} h(x)-\frac{\rho}{2}\|A x-b\|^{2}+\theta(z)+\frac{\rho}{2}\|z\|^{2} \\
\text { s.t. } \quad A x+z=b,
\end{array}
$$

where $\rho \in\left(0, \frac{\sigma_{h}}{\|A\|^{2}}\right)$. Let

$$
f(x)=h(x)-\frac{\rho}{2}\|A x-b\|^{2}, g(z)=\theta(z)+\frac{\rho}{2}\|z\|^{2} .
$$

Then, $f$ is strongly convex with modulus $\sigma_{h}-\rho\|A\|^{2}$, and $g$ is strongly convex with modulus $\rho$. Then, (4.4) has the form of (1.1) and satisfies the assumption $(\mathcal{P})$.

The Lagrangian function of (4.4) is

$$
\begin{aligned}
\hat{\mathcal{L}}(x, z, y)= & h(x)-\frac{\rho}{2}\|A x-b\|^{2}+\theta(z)+\frac{\rho}{2}\|z\|^{2}+ \\
& \langle y, b-A x-z\rangle .
\end{aligned}
$$

If $\left(x^{*}, z^{*}, y^{*}\right)$ is a saddle point of the Lagrangian function $\hat{\mathcal{L}}(\cdot)$, then

$$
\left\{\begin{array}{c}
A^{*}\left(y^{*}-\rho z^{*}\right) \in \partial h\left(x^{*}\right) \\
y^{*}-\rho z^{*} \in \partial \theta\left(z^{*}\right) \\
A x^{*}+z^{*}-b=0 .
\end{array}\right.
$$

That is, $\left(x^{*}, z^{*}, y^{*}-\rho z^{*}\right)$ is a saddle point of the Lagrangian function of (4.3). For concreteness, we present the dynamical alternating direction method of multipliers for (4.4) implicitly below:

$$
\left\{\begin{array}{c}
\dot{x}(t)+x(t) \in\left(\partial h+(c(t)-\rho) A^{*} A\right)^{-1}\left(A^{*} y(t)-\right. \\
\left.\rho A^{*} b-c(t) A^{*} z(t)+c(t) A^{*} b\right) \\
\dot{z}(t)+z(t) \in(\partial \theta+c(t)+\rho)^{-1}(y(t)-c(t) A(\dot{x}(t) \\
+x(t))+c(t) b) \\
\dot{y}(t)=\sigma c(t)(b-A(\dot{x}(t)+x(t))-(\dot{z}(t)+z(t))) \\
x(0)=x^{0} \in \mathcal{H}, z(0)=z^{0} \in \mathcal{G}, y(0)=y^{0} \in \mathcal{K},
\end{array}\right.
$$

where $\sigma>0, c(t)>0$ for all $t \in[0,+\infty), \rho \in\left(0, \frac{\sigma_{h}}{\|A\|^{2}}\right)$.

In this section, we assume that $s(t):=(x(t), z(t), y(t))$ $:[0,+\infty) \rightarrow \mathcal{H} \times \mathcal{G} \times \mathcal{K}$ be the trajectory of the dynamical system $(4.6)$.

Remark 5 It is easy to see that the dynamical alternating direction method of multipliers for (4.3) is defined implicitly as follows:

$$
\left\{\begin{aligned}
& \dot{x}(t)+x(t) \in\left(\partial h+c(t) A^{*} A\right)^{-1}\left(A^{*} y(t)-c(t) A^{*} z(t)\right. \\
&\left.+c(t) A^{*} b\right) \\
& \dot{z}(t)+z(t) \in(\partial \theta+c(t))^{-1}(y(t)-c(t) A(\dot{x}(t)+x(t)) \\
&+c(t) b) \\
& \dot{y}(t)=\sigma c(t)(b-A(\dot{x}(t)+x(t))-(\dot{z}(t)+z(t))) \\
& x(0)=x^{0} \in \mathcal{H}, z(0)=z^{0} \in \mathcal{G}, y(0)=y^{0} \in \mathcal{K},
\end{aligned}\right.
$$

By comparison, it is easy to see that (4.6) is very similar to (4.7).

Similar with Theorems 2, we can obtain the following convergence results of the dynamical alternating direction method of multipliers (4.6): 
Corollary 1 In the setting of the optimization problem (4.3), assume that the set of saddle points of the Lagrangian $\mathcal{L}$ is nonempty, $\rho \in\left(0, \frac{\sigma_{h}}{\|A\|^{2}}\right)$, for $0<\epsilon<$ $\frac{1}{2} \min \left\{\frac{\sigma_{h}}{\|A\|^{2}}-\rho, \rho\right\}$ the function $c:[0,+\infty) \rightarrow$ $\left[\epsilon, \min \left\{\frac{\sigma_{h}}{\|A\|^{2}}-\rho, \rho\right\}-\epsilon\right]$ is Lipschitz continuous and monotonically decreasing. If $c(t)$ is a constant function, namely $c(t)=c$ for all $t \in[0,+\infty)$, then it is enough to assume that $\epsilon \leq c \leq \min \left\{\frac{2 \sigma_{h}}{\|A\|^{2}}-2 \rho, \rho\right\}-\epsilon$. For an arbitrary starting point $\left(x^{0}, z^{0}, y^{0}\right) \in \mathcal{H} \times \mathcal{G} \times \mathcal{K}$, let $(x, z, y)$ : $[0,+\infty) \rightarrow \mathcal{H} \times \mathcal{G} \times \mathcal{K}$ be the unique strong global solution of the dynamical system (4.6). Then the trajectory $(x(t), z(t), y(t))$ converges weakly to a saddle point of $\hat{\mathcal{L}}$ as $t \rightarrow \infty$. If $\lim _{t \rightarrow+\infty} s(t)=\left(x^{*}, z^{*}, y^{*}\right)$, then $\left(x^{*}, z^{*}, y^{*}-\rho z^{*}\right)$ is the saddle point of the Lagrangian function of (4.3).

\section{Conclusions}

In this paper, we introduce and study a dynamical system for solving the two-block separable strongly convex minimization problem with linear constraints. In the framework of Lyapunov analysis, by finding an appropriate energy functional, we prove that the solution trajectory of the system converges to the saddle point of Lagrange function of the problem. Furthermore, when the coefficient matrices in the constraint are identiy matrices, we prove the worst-case $O\left(\frac{1}{t}\right)$ convergence rate in ergodic sense. In addition, we demonstrate how the dynamical alternating direction method of multipliers can be applied in the absence of a strongly convex block. The discretization of the dynamical system considered is the classical ADMM.

\section{A . Proof of Section 3}

\section{Lemma 1}

Proof Let $t \in[0,+\infty)$ be fixed. Then we have

$$
0 \in \partial\left(f(\cdot)+\frac{c(t)}{2}\|A \cdot\|^{2}-c(t)\langle\cdot, u\rangle\right)\left(K_{t}(u)\right) .
$$

For all $u, v \in \mathcal{H}$ we obtain

$$
c(t) u \in \partial f\left(K_{t}(u)\right)+\left(c(t) A^{*} A\right)\left(K_{t}(u)\right)
$$

and

$$
c(t) v \in \partial f\left(K_{t}(v)\right)+\left(c(t) A^{*} A\right)\left(K_{t}(v)\right) .
$$

Since $\partial f+c(t) A^{*} A$ is $\sigma_{f}$-strongly monotone, we have

$$
\sigma_{f}\left\|K_{t} u-K_{t} v\right\|^{2} \leq c(t)\left\langle u-v, K_{t}(u)-K_{t}(v)\right\rangle .
$$

Using the Cauchy-Schwarz inequality it follows

$$
\left\|K_{t} u-K_{t} v\right\| \leq \frac{c(t)}{\sigma_{f}}\|u-v\|
$$

which means that $K_{t}$ is $\frac{c(t)}{\sigma_{f}}$-Lipschitz continuous.

For $t \in[0,+\infty)$ fixed we have

$$
0 \in \partial\left(g(\cdot)+\frac{c(t)}{2}\|B \cdot\|^{2}-c(t)\langle\cdot, v\rangle\right)\left(J_{t}(v)\right) .
$$

For all $u, v \in \mathcal{G}$ we obtain

$$
c(t) u \in \partial g\left(J_{t}(u)\right)+\left(c(t) B^{*} B\right)\left(J_{t}(u)\right)
$$

and

$$
c(t) v \in \partial g\left(J_{t}(v)\right)+\left(c(t) B^{*} B\right)\left(J_{t}(v)\right) .
$$

Since $\partial g+c(t) B^{*} B$ is $\sigma_{g}$-strongly monotone, we have

$$
\sigma_{g}\left\|J_{t} u-J_{t} v\right\|^{2} \leq c(t)\left\langle u-v, J_{t}(u)-J_{t}(v)\right\rangle .
$$

Using the Cauchy-Schwarz inequality it follows

$$
\left\|J_{t} u-J_{t} v\right\| \leq \frac{c(t)}{\sigma_{g}}\|u-v\|
$$

which means that $J_{t}$ is $\frac{c(t)}{\sigma_{g}}$-Lipschitz continuous.

\section{Lemma 2}

Proof Let $t, r \in[0, \infty)$ be fixed.

(i)Form the definition of $R_{(x, z, y)}$ we have

$$
\begin{aligned}
& A^{*} y-c(t) A^{*} B z+c(t) A^{*} b \\
& \in \partial f\left(R_{(x, z, y)}(t)+x\right)+c(t) A^{*} A\left(R_{(x, z, y)}(t)+x\right)
\end{aligned}
$$

and

$$
\begin{aligned}
& A^{*} y-c(r) A^{*} B z+c(r) A^{*} b \\
& \in \partial f\left(R_{(x, z, y)}(r)+x\right)+c(r) A^{*} A\left(R_{(x, z, y)}(r)+x\right) .
\end{aligned}
$$

If we add $c(t) A^{*} A\left(R_{(x, z, y)}(r)+x\right)$ on both sides of the relation above, we have

$$
\begin{aligned}
& (c(t)-c(r)) A^{*} A\left(R_{(x, z, y)}(r)+x\right)+A^{*} y-c(r) A^{*} B z \\
& +c(r) A^{*} b \\
& \in \partial f\left(R_{(x, z, y)}(r)+x\right)+c(t) A^{*} A\left(R_{(x, z, y)}(r)+x\right) .
\end{aligned}
$$

From(A.2) and (A.3) and using that $\partial f+c(t) A^{*} A$ is $\sigma_{f}$-strongly monotone, we have

$$
\begin{aligned}
& \left\langle(c(t)-c(r)) A^{*}\left[A\left(R_{(x, z, y)}(r)+x\right)+B z-b\right],\right. \\
& \left.R_{(x, z, y)}(r)-R_{(x, z, y)}(t)\right\rangle \\
& \geq \sigma_{f}\left\|R_{(x, z, y)}(r)-R_{(x, z, y)}(t)\right\|^{2} .
\end{aligned}
$$


Form the Cauchy-Schwarz inequality, the definition of $P_{(x, z, y)}$ it follows

$$
\begin{aligned}
&\left\|R_{(x, z, y)}(t)-R_{(x, z, y)}(r)\right\| \\
& \leq \frac{1}{\sigma_{f}} \|(c(t)-c(r)) A^{*}(A x+B z-b) \\
&+(c(t)-c(r)) A^{*} A R_{(x, z, y)}(r) \| \\
&= \frac{1}{\sigma_{f}} \|(c(t)-c(r)) A^{*}\left(-A R_{(x, z, y)}(r)-B Q_{(x, z, y)}(r)\right. \\
&\left.-\frac{1}{\sigma c(r)} P_{(x, z, y)}(r)\right)+(c(t)-c(r)) A^{*} A R_{(x, z, y)}(r) \| \\
&= \frac{1}{\sigma_{f}} \|-(c(t)-c(r)) A^{*} B Q_{(x, z, y)}(r) \\
&-\frac{c(t)-c(r)}{\sigma c(r)} A^{*} P_{(x, z, y)}(r) \| \\
& \leq\left(\frac{\|A\|\|B\|\left\|Q_{(x, z, y)}(r)\right\|}{\sigma_{f}}\right. \\
&\left.+\frac{\|A\|\left\|P_{(x, z, y)}(r)\right\|}{\sigma \sigma_{f} c(r)}\right)|c(t)-c(r)| .
\end{aligned}
$$

(ii)Form the definition of $Q_{(x, z, y)}$ we have

$$
\begin{aligned}
& B^{*} y-c(t) B^{*} A\left(R_{(x, z, y)}(t)+x\right)+c(t) B^{*} b \\
& \in \partial g\left(Q_{(x, z, y)}(t)+z\right)+c(t) B^{*} B\left(Q_{(x, z, y)}(t)+z\right)
\end{aligned}
$$

and

$$
\begin{aligned}
& B^{*} y-c(r) B^{*} A\left(R_{(x, z, y)}(r)+x\right)+c(r) B^{*} b \\
& \in \partial g\left(Q_{(x, z, y)}(r)+z\right)+c(r) B^{*} B\left(Q_{(x, z, y)}(r)+z\right) .
\end{aligned}
$$

If we add $c(t) B^{*} B\left(Q_{(x, z, y)}(r)+z\right)$ on both sides of the relation above, we have

$$
\begin{aligned}
& (c(t)-c(r)) B^{*} B\left(Q_{(x, z, y)}(r)+z\right)+B^{*} y \\
& -c(r) B^{*} A\left(R_{(x, z, y)}(r)+x\right)+c(r) B^{*} b \\
& \in \partial g\left(Q_{(x, z, y)}(r)+z\right)+c(t) B^{*} B\left(Q_{(x, z, y)}(r)+z\right) .
\end{aligned}
$$

From(A.4) and (A.5) and using that $\partial g+c(t) B^{*} B$ is $\sigma_{g}$-strongly monotone, we have

$$
\begin{aligned}
& \left\langle(c(t)-c(r)) B^{*} B\left(Q_{(x, z, y)}(r)+z\right)+(c(t)-c(r)) B^{*} A x\right. \\
& +c(t) B^{*} A R_{(x, z, y)}(t)-c(r) B^{*} A R_{(x, z, y)}(r) \\
& \left.-(c(t)-c(r)) B^{*} b, Q_{(x, z, y)}(r)-Q_{(x, z, y)}(t)\right\rangle \\
& \geq \sigma_{g}\left\|Q_{(x, z, y)}(r)-Q_{(x, z, y)}(t)\right\|^{2} .
\end{aligned}
$$

Form the Cauchy-Schwarz inequality, the definition of $P_{(x, z, y)}$ and (i) it follows

$$
\begin{aligned}
& \left\|Q_{(x, z, y)}(t)-Q_{(x, z, y)}(r)\right\| \\
& \leq \frac{1}{\sigma_{g}} \|(c(t)-c(r)) B^{*}(A x+B z-b)
\end{aligned}
$$

$$
\begin{aligned}
& +(c(t)-c(r)) B^{*} B Q_{(x, z, y)}(r)+c(t) B^{*} A R_{(x, z, y)}(t) \\
& -c(r) B^{*} A R_{(x, z, y)}(r) \| \\
= & \frac{1}{\sigma_{g}} \|(c(t)-c(r)) B^{*}\left(-A R_{(x, z, y)}(r)-B Q_{(x, z, y)}(r)\right. \\
& \left.-\frac{1}{\sigma c(r)} P_{(x, z, y)}(r)\right)+(c(t)-c(r)) B^{*} B Q_{(x, z, y)}(r) \\
& +c(t) B^{*} A R_{(x, z, y)}(t)-c(r) B^{*} A R_{(x, z, y)}(r) \| \\
= & \frac{1}{\sigma_{g}} \| c(t) B^{*} A\left(R_{(x, z, y)}(t)-R_{(x, z, y)}(r)\right) \\
& -\frac{c(t)-c(r)}{\sigma c(r)} B^{*} P_{(x, z, y)}(r) \| \\
\leq & \frac{c(t)}{\sigma_{g}}\|A\|\|B\|\left\|R_{(x, z, y)}(t)-R_{(x, z, y)}(r)\right\| \\
& +\frac{|c(t)-c(r)|_{\|B\|\left\|P_{(x, z, y)}(r)\right\|}^{\sigma c(r) \sigma_{g}} \|}{\sigma \sigma_{f} \sigma_{g} c(r)} \\
\leq & \left(\frac{c(t)\|A\|^{2}\|B\|^{2}\left\|Q_{(x, z, y)}(r)\right\|}{\sigma_{f} \sigma_{g}}\right. \\
& \left.+\frac{\left(c(t)\|A\|^{2}+\sigma_{f}\right)\|B\|\left\|P_{(x, z, y)}(r)\right\|}{\sigma}\right)|c(t)-c(r)| .
\end{aligned}
$$

\section{Theorem 1}

Proof In the following we use the equivalent formulation of the dynamical system described in Remark 4. We show the existence and uniqueness of a strong global solution using the Cauchy-Lipschitz-Picard Theorem.

In the first part we have to show, that $\Gamma(t, \cdot, \cdot, \cdot)$ is $L(t)$-Lipschitz continuous for every $t \in[0,+\infty)$ and that the Lipschitz constant as a function of time fulfills $L(\cdot) \in L_{l o c}^{1}([0,+\infty), \Re)$. In the second part we will prove that $\Gamma(\cdot, x, z, y) \in L_{l o c}^{1}([0,+\infty), \mathcal{H} \times \mathcal{G} \times \mathcal{K})$ for every $(x, z, y) \in \mathcal{H} \times \mathcal{G} \times \mathcal{K}$.

(1)Let $t \in[0,+\infty)$ be fixed and let $(x, z, y),(\bar{x}, \bar{z}, \bar{y}) \in$ $\mathcal{H} \times \mathcal{G} \times \mathcal{K}$. We have

$$
\begin{aligned}
& \|\Gamma(t, x, z, y)-\Gamma(t, \bar{x}, \bar{z}, \bar{y})\| \\
& =\sqrt{\|u-\bar{u}\|^{2}+\|v-\bar{v}\|^{2}+\|w-\bar{w}\|^{2}}
\end{aligned}
$$

where (taking into account Lemma 1)

$$
\begin{aligned}
u- & \bar{u} \\
= & \underset{p \in \mathcal{H}}{\operatorname{argmin}}\left\{F(t, p)+\frac{c(t)}{2}\left\|p-\left(\frac{1}{c(t)} A^{*} y-A^{*} B z+A^{*} b\right)\right\|^{2}\right\} \\
& -\underset{p \in \mathcal{H}}{\operatorname{argmin}}\left\{F(t, p)+\frac{c(t)}{2}\left\|p-\left(\frac{1}{c(t)} A^{*} \bar{y}-A^{*} B \bar{z}+A^{*} b\right)\right\|^{2}\right\} \\
& +\bar{x}-x \\
= & K_{t}\left(\frac{1}{c(t)} A^{*} y-A^{*} B z+A^{*} b\right)-K_{t}\left(\frac{1}{c(t)} A^{*} \bar{y}-A^{*} B \bar{z}+A^{*} b\right) \\
& +\bar{x}-x .
\end{aligned}
$$

Therefore,

$\|u-\bar{u}\|^{2}$ 


$$
\begin{aligned}
& \begin{aligned}
\leq & 2\left\|K_{t}\left(\frac{1}{c(t)} A^{*} y-A^{*} B z+A^{*} b\right)-K_{t}\left(\frac{1}{c(t)} A^{*} \bar{y}-A^{*} B \bar{z}+A^{*} b\right)\right\|^{2} \leq \frac{8 c^{2}(t)}{\sigma_{g}^{2}}\|A\|^{2}\|B\|^{2}\|u-\bar{u}\|^{2}+\frac{8 c^{2}(t)}{\sigma_{g}^{2}}\|A\|^{2}\|B\|^{2}\|x-\bar{x}\|^{2} \\
& +2\|\bar{x}-x\|^{2} .
\end{aligned} \\
& \text { Form Lemma } 1 \text { we know, that } K_{t} \text { is } \frac{c(t)}{\sigma_{f}} \text {-Lipschitz con- } \\
& \text { tinuous. Thus: } \\
& \begin{aligned}
&\|u-\bar{u}\|^{2} \\
& \leq \frac{2 c^{2}(t)}{\sigma_{f}^{2}}\left\|-A^{*} B(z-\bar{z})+\frac{1}{c(t)} A^{*}(y-\bar{y})\right\|^{2}+2\|\bar{x}-x\|^{2} \\
& \leq \frac{2 c^{2}(t)}{\sigma_{f}^{2}}\left(2\left\|A^{*} B(z-\bar{z})\right\|^{2}+2\left\|\frac{1}{c(t)} A^{*}(y-\bar{y})\right\|^{2}\right) \\
&+2\|\bar{x}-x\|^{2} \\
& \leq \frac{2 c^{2}(t)}{\sigma_{f}^{2}}\left(2\|A\|^{2}\|B\|^{2}\|z-\bar{z}\|^{2}+\frac{2}{c^{2}(t)}\|A\|^{2}\|y-\bar{y}\|^{2}\right) \\
&+2\|x-\bar{x}\|^{2} \\
&= 2\|x-\bar{x}\|^{2}+\frac{4 c^{2}(t)}{\sigma_{f}^{2}}\|A\|^{2}\|B\|^{2}\|z-\bar{z}\|^{2} \\
&+\frac{4}{\sigma_{f}^{2}}\|A\|^{2}\|y-\bar{y}\|^{2} .
\end{aligned} \\
& +2\|\bar{z}-z\|^{2}+\frac{4}{\sigma_{g}^{2}}\|B\|^{2}\|y-\bar{y}\|^{2} \\
& \leq \frac{24 c^{2}(t)}{\sigma_{g}^{2}}\|A\|^{2}\|B\|^{2}\|x-\bar{x}\|^{2} \\
& +\left(\frac{32 c^{4}(t)}{\sigma_{f}^{2} \sigma_{g}^{2}}\|A\|^{4}\|B\|^{4}+2\right)\|z-\bar{z}\|^{2} \\
& +\left(\frac{4\|B\|^{2}}{\sigma_{g}^{2}}+\frac{32 c^{2}(t)\|A\|^{4}\|B\|^{2}}{\sigma_{f}^{2} \sigma_{g}^{2}}\right)\|y-\bar{y}\|^{2} .
\end{aligned}
$$

Finally,

$$
\begin{aligned}
& \|w-\bar{w}\|^{2} \\
& =\|-\sigma c(t)(A(u-\bar{u}+x-\bar{x})+B(v-\bar{v}+z-\bar{z}))\|^{2} \\
& \leq 4 \sigma^{2} c^{2}(t)\|A\|^{2}\|u-\bar{u}\|^{2}+4 \sigma^{2} c^{2}(t)\|A\|^{2}\|x-\bar{x}\|^{2} \\
& +4 \sigma^{2} c^{2}(t)\|B\|^{2}\|v-\bar{v}\|^{2}+4 \sigma^{2} c^{2}(t)\|B\|^{2}\|z-\bar{z}\|^{2} \\
& \leq 12 \sigma^{2} c^{2}(t)\|A\|^{2}\left(\frac{8 c^{2}(t)\|B\|^{4}}{\sigma_{g}^{2}}+1\right)\|x-\bar{x}\|^{2} \\
& +4 \sigma^{2} c^{2}(t)\|B\|^{2}\left(\frac{4 c^{2}(t)\|A\|^{4}}{\sigma_{f}^{2}}+\frac{32 c^{4}(t)\|A\|^{4}\|B\|^{4}}{\sigma_{f}^{2} \sigma_{g}^{2}}\right. \\
& \text { +3) }\|z-\bar{z}\|^{2}+4 \sigma^{2} c^{2}(t)\left(\frac{4\|A\|^{4}}{\sigma_{f}^{2}}+\frac{4\|B\|^{4}}{\sigma_{g}^{2}}\right. \\
& \left.+\frac{32 c^{2}(t)\|A\|^{4}\|B\|^{4}}{\sigma_{f}^{2} \sigma_{g}^{2}}\right)\|y-\bar{y}\|^{2} .
\end{aligned}
$$

Then we have,

$$
\begin{aligned}
& \|\Gamma(t, x, z, y)-\Gamma(t, \bar{x}, \bar{z}, \bar{y})\| \\
& \leq \sqrt{L_{1}(t)\|x-\bar{x}\|^{2}+L_{2}(t)\|z-\bar{z}\|^{2}+L_{3}(t)\|y-\bar{y}\|^{2}} \\
& \leq \sqrt{L_{1}(t)+L_{2}(t)+L_{3}(t)} \sqrt{\|x-\bar{x}\|^{2}+\|z-\bar{z}\|^{2}+\|y-\bar{y}\|^{2}} \\
& =L(t)\|(x, z, y)-(\bar{x}, \bar{z}, \bar{y})\|,
\end{aligned}
$$

According to Lemma 1, we have that $J_{t}$ is $\frac{c(t)}{\sigma_{g}}$-Lipschitz continuous. We derive:

where

$$
L(t)=\sqrt{L_{1}(t)+L_{2}(t)+L_{3}(t)},
$$

$\|v-\bar{v}\|^{2}$

$$
\begin{aligned}
\leq & 2 \| J_{t}\left(\frac{1}{c(t)} B^{*} y-B^{*} A(u+x)+B^{*} b\right) \\
& -J_{t}\left(\frac{1}{c(t)} B^{*} \bar{y}-B^{*} A(\bar{u}+\bar{x})+B^{*} b\right)\left\|^{2}+2\right\| \bar{z}-z \|^{2} \\
\leq & \frac{2 c^{2}(t)}{\sigma_{g}^{2}}\left\|B^{*} A(\bar{x}-x+\bar{u}-u)+\frac{1}{c(t)} B^{*}(y-\bar{y})\right\|^{2} \\
& +2\|\bar{z}-z\|^{2} \\
\leq & \frac{2 c^{2}(t)}{\sigma_{g}^{2}}\left(2\|B\|^{2}\|A\|^{2}\|\bar{x}-x+\bar{u}-u\|^{2}\right. \\
& \left.+\frac{2}{c^{2}(t)}\|B\|^{2}\|y-\bar{y}\|^{2}\right)+2\|\bar{z}-z\|^{2}
\end{aligned}
$$

and

$$
\begin{aligned}
L_{1}(t)= & 2+12 \sigma^{2} c^{2}(t)\|A\|^{2}+\frac{24 c^{2}(t)\left(4 \sigma^{2} c^{2}(t)\|B\|^{2}+1\right)}{\sigma_{g}^{2}}\|A\|^{2}\|B\|^{2}, \\
L_{2}(t)= & 2+12 \sigma^{2} c^{2}(t)\|B\|^{2}+\frac{4 c^{2}(t)\left(4 \sigma^{2} c^{2}(t)\|A\|^{2}+1\right)}{\sigma_{f}^{2}}\|A\|^{2}\|B\|^{2} \\
& +\frac{32 c^{4}(t)\left(4 \sigma^{2} c^{2}(t)+1\right)}{\sigma_{f}^{2} \sigma_{g}^{2}}\|A\|^{4}\|B\|^{4}, \\
L_{3}(t)= & \frac{4\left(4 \sigma^{2} c^{2}(t)\|A\|^{2}+1\right)}{\sigma_{f}^{2}}\|A\|^{2}+\frac{4\left(4 \sigma^{2} c^{2}(t)+1\right)}{\sigma_{g}^{2}}\|B\|^{2} \\
& +\frac{32 c^{2}(t)\left(4 \sigma^{2} c^{2}(t)+1\right)}{\sigma_{f}^{2} \sigma_{g}^{2}}\|A\|^{4}\|B\|^{2},
\end{aligned}
$$


which means that $\Gamma(t, \cdot, \cdot, \cdot)$ is $L(t)$-Lipschitz continuous. Since $c(t)$ is bounded, it follows that $L(\cdot) \in L_{l o c}^{1}$ $([0,+\infty), \Re)$.

(2)Now we will show that $\Gamma(\cdot, x, z, y) \in L_{l o c}^{1}([0,+\infty)$, $\mathcal{H} \times \mathcal{G} \times \mathcal{K})$ for every $(x, z, y) \in \mathcal{H} \times \mathcal{G} \times \mathcal{K}$. Let $(x, z, y) \in$ $\mathcal{H} \times \mathcal{G} \times \mathcal{K}$ be fixed and $T>0$. We have

$\int_{0}^{T}\|\Gamma(t, x, z, y)\| d t$

$=\int_{0}^{T} \sqrt{\|u(t, x, z, y)\|^{2}+\|v(t, x, z, y)\|^{2}+\|w(t, x, z, y)\|^{2}} d t$.

Form Lemma 2, we have

$$
\begin{array}{rl}
\|u(t, x, z, y)\|^{2} & 2\|u(t, x, z, y)-u(0, x, z, y)\|^{2}+2\|u(0, x, z, y)\|^{2} \\
\leq & 2\left[\left(\frac{\|A\|\|B\|\|v(0, x, z, y)\|}{\sigma_{f}}+\right.\right. \\
& \left.\left.\quad \frac{\|A\|\|w(0, x, z, y)\|}{\sigma \sigma_{f} c(0)}\right)|c(t)-c(0)|\right]^{2}+2\|u(0, x, z, y)\|^{2} \\
\leq & \frac{4\|A\|^{2}}{\sigma_{f}^{2}}\left(\|B\|^{2}\|v(0, x, z, y)\|^{2}\right. \\
& \left.+\frac{\|w(0, x, z, y)\|^{2}}{\sigma^{2} c^{2}(0)}\right)|c(t)-c(0)|^{2}+2\|u(0, x, z, y)\|^{2} \\
\|v(t, x, z, y)\|^{2} & \\
\leq & 2\|v(t, x, z, y)-v(0, x, z, y)\|^{2}+2\|v(0, x, z, y)\|^{2} \\
\leq & 2\left[\left(\frac{c(t)\|A\|^{2}\|B\|^{2}\|v(0, x, z, y)\|}{\sigma_{f} \sigma_{g}}\right.\right. \\
& +2\|v(0, x, z, y)\|^{2}, \\
& \left.\left.+\frac{\left(c(t)\|A\|^{2}+\sigma_{f}\right)\|B\|\|w(0, x, z, y)\|}{\sigma \sigma_{f} \sigma_{g} c(0)}\right)|c(t)-c(0)|\right]^{2} \\
& +2\|v(0, x, z, y)\|^{2} \\
\leq & \frac{4\|B\|^{2}}{\sigma_{f}^{2} \sigma_{g}^{2}}\left(c(t)\|A\|^{4}\|B\|^{2}\|v(0, x, z, y)\|^{2}\right. \\
+ & \left.\frac{\left(c(t)\|A\|^{2}+\sigma_{f}\right)^{2}\|w(0, x, z, y)\|^{2}}{\sigma^{2} c^{2}(0)}\right)|c(t)-c(0)|^{2} \\
& \\
& \\
&
\end{array}
$$

and

$$
\begin{aligned}
& \|w(t, x, z, y)\|^{2} \\
& \leq \sigma^{2} c^{2}(t)\|b-A(u(t, x, z, y)+x)-B(v(t, x, z, y)+z)\|^{2} \\
& \leq 4 \sigma^{2} c^{2}(t)\left(\|b\|^{2}+\|A\|^{2}\|u(0, x, z, y)\|^{2}\right. \\
& \left.\quad+\|B\|^{2}\|v(0, x, z, y)\|^{2}+\|A x+B z\|^{2}\right) .
\end{aligned}
$$

Since $c(t)$ is bounded, it follows that the integral

$$
\int_{0}^{T}\|\Gamma(t, x, z, y)\| d t
$$

exists and it is finite. So we have that $\Gamma(\cdot, x, z, y) \in$ $L_{\text {loc }}^{1}([0,+\infty), \mathcal{H} \times \mathcal{G} \times \mathcal{K})$. The conclusion follows.

\section{B . Proof of Section 4}

\section{Lemma 3}

Proof Let $T>0$ be fixed. In the following we use the notation (2.3) again. Further, for the sake of brevity of the following proof, we note that $u(t, t)=u(t, x(t), z(t), y(t))$, $u(t, r)=u(t, x(r), z(r), y(r)), v(t, t)=v(t, x(t), z(t), y(t)), v(t, r)=$ $v(t, x(r), z(r), y(r)), w(t, t)=w(t, x(t), z(t), y(t))$, and $w(t, r)=$ $w(t, x(r), z(r), y(r))$. Let $t, r \in[0, T]$ be fixed. We have

$$
\begin{aligned}
& \|\dot{U}(t)-\dot{U}(r)\| \\
& =\|\Gamma(t, U(t))-\Gamma(r, U(r))\| \\
& \leq\|\Gamma(t, U(t))-\Gamma(t, U(r))\|+\|\Gamma(t, U(r))-\Gamma(r, U(r))\| \\
& \leq\|u(t, t)-u(t, r)\|+\|v(t, t)-v(t, r)\| \\
& \quad+\|w(t, t)-w(t, r)\|+\|u(t, r)-u(r, r)\| \\
& \quad+\| v(t, r)-v(r, r))\|+\| w(t, r)-w(r, r) \| .
\end{aligned}
$$

Since

$$
\begin{aligned}
u(t, t)-u(t, r) & \\
= & K_{t}\left(\frac{1}{c(t)} A^{*} y(t)-A^{*} B z(t)+A^{*} b\right) \\
& -K_{t}\left(\frac{1}{c(t)} A^{*} y(r)-A^{*} B z(r)+A^{*} b\right)-x(t)+x(r),
\end{aligned}
$$

from Lemma 1 we get

$$
\begin{aligned}
& \|u(t, t)-u(t, r)\| \\
& \leq \frac{c(t)}{\sigma_{f}}\left\|\frac{1}{c(t)} A^{*}(y(t)-y(r))-A^{*} B(z(t)-z(r))\right\| \\
& \quad+\|x(t)-x(r)\| \\
& \leq\|x(t)-x(r)\|+\frac{c(t)\|A\|\|B\|}{\sigma_{f}}\|z(t)-z(r)\| \\
& \quad+\frac{\|A\|}{\sigma_{f}}\|y(t)-y(r)\| .
\end{aligned}
$$

Since $t \rightarrow c(t)$ is bounded on $[0, T]$, there exists $L_{1}:=$ $L_{1}(T)>0$ such that

$$
\begin{aligned}
& \|u(t, t)-u(t, r)\| \\
& \leq L_{1}(\|x(t)-x(r)\|+\|z(t)-z(r)\|+\|y(t)-y(r)\|) .
\end{aligned}
$$

Similarly we have

$$
\begin{aligned}
& v(t, t)-v(t, r) \\
& =J_{t}\left(\frac{1}{c(t)} B^{*} y(t)-B^{*} A(u(t, t)+x(t))+B^{*} b\right) \\
& \quad-J_{t}\left(\frac{1}{c(t)} B^{*} y(r)-B^{*} A(u(t, r)+x(r))+B^{*} b\right) \\
& \quad-z(t)+z(r),
\end{aligned}
$$




$$
\begin{aligned}
& \|v(t, t)-v(t, r)\| \\
& \leq \frac{c(t)}{\sigma_{f}} \| \frac{1}{c(t)} B^{*}(y(t)-y(r))-B^{*} A(x(t)-x(r)) \\
& \quad-B^{*} A(u(t, t)-u(t, r)\|+\| z(t)-z(r) \| \\
& \leq \frac{c(t)\|A\|\|B\|}{\sigma_{f}}\|x(t)-x(r)\|+\|z(t)-z(r)\| \\
& \quad+\frac{\|B\|}{\sigma_{f}}\|y(t)-y(r)\|+\frac{c(t)\|A\|\|B\|}{\sigma_{f}}\|u(t, t)-u(t, r)\| \\
& \leq \\
& \quad \frac{c(t)\|A\|\|B\|\left(L_{1}+1\right)}{\sigma_{f}}\|x(t)-x(r)\| \\
& \quad+\left(\frac{c(t)\|A\|\|B\| L_{1}}{\sigma_{f}}+1\right)\|z(t)-z(r)\| \\
& \quad+\frac{\|B\|\left(c(t)\|A\| L_{1}+1\right)}{\sigma_{f}}\|y(t)-y(r)\| .
\end{aligned}
$$

Since $t \rightarrow c(t)$ is bounded on $[0, T]$, there exists $L_{2}:=$ $L_{2}(T)>0$ such that

$$
\begin{aligned}
& \|v(t, t)-v(t, r)\| \\
& \leq L_{2}(\|x(t)-x(r)\|+\|z(t)-z(r)\|+\|y(t)-y(r)\|) .
\end{aligned}
$$

Using (B.1) and (B.2) we obtain

$$
\begin{aligned}
& \|w(t, t)-w(t, r)\| \\
& \leq \sigma c(t)\|A\|\|u(t, t)-u(t, r)+x(t)-x(r)\| \\
& +\sigma c(t)\|B\|\|v(t, t)-v(t, r)+z(t)-z(r)\| \\
& \leq \sigma c(t)\left(\|A\| L_{1}+\|B\| L_{2}+\|A\|\right)\|x(t)-x(r)\| \\
& +\sigma c(t)\left(\|A\| L_{1}+\|B\| L_{2}+\|B\|\right)\|z(t)-z(r)\| \\
& +\sigma c(t)\left(\|A\| L_{1}+\|B\| L_{2}\right)\|y(t)-y(r)\| \text {. }
\end{aligned}
$$

So there exists $L_{3}:=L_{3}(T)>0$ such that

$$
\begin{aligned}
& \|w(t, t)-w(t, r)\| \\
& \leq L_{3}(\|x(t)-x(r)\|+\|z(t)-z(r)\|+\|y(t)-y(r)\|) .
\end{aligned}
$$

Using Lemma 2(i), we obtain

$$
\begin{aligned}
& \|u(t, r)-u(r, r)\| \\
& =\left\|R_{(x(r), z(r), y(r))}(t)-R_{(x(r), z(r), y(r))}(r)\right\| \\
& \leq\left(\frac{\|A\|\|B\|\left\|Q_{(x(r), z(r), y(r))}(r)\right\|}{\sigma_{f}}\right. \\
& \left.\quad+\frac{\|A\|\left\|P_{(x(r), z(r), y(r))}(r)\right\|}{\sigma \sigma_{f} c(r)}\right)|c(t)-c(r)| .
\end{aligned}
$$

Since $r \mapsto K_{r}, r \mapsto J_{r}$ and $x, z, y$ and $M_{1}$ are absolutely continuous, the maps

$$
\begin{aligned}
r \mapsto Q_{(x(r), z(r), y(r))}(r)= & J_{r}\left(\frac{B^{*}}{c(r)} y(r)-B^{*} A(u(r, r)\right. \\
& \left.\left.+x(r))+B^{*} b\right)\right)-z(r)
\end{aligned}
$$

and

$$
\begin{aligned}
r \mapsto P_{(x(r), z(r), y(r))}(r)= & \sigma c(r)\left(b-A\left(R_{(x(r), z(r), y(r))}(r)\right.\right. \\
& +x(r))-B\left(Q_{(x(r), z(r), y(r))}(r)\right. \\
& +z(r)))
\end{aligned}
$$

are bounded in $[0, T]$. Therefore, there exists $L_{4}:=$ $L_{4}(T)>0$ such that

$\|u(t, r)-u(r, r)\| \leq L_{4}|c(t)-c(r)|$.

In an analog way, using Lemma 2(ii), we get

$$
\begin{aligned}
& \|v(t, r)-v(r, r)\| \\
& =\left\|Q_{(x(r), z(r), y(r))}(t)-Q_{(x(r), z(r), y(r))}(r)\right\| \\
& \leq\left(\frac{c(t)\|A\|^{2}\|B\|^{2}\left\|Q_{(x(r), z(r), y(r))}(r)\right\|}{\sigma_{f} \sigma_{g}}+\right. \\
& \left.\frac{\left(c(t)\|A\|^{2}+\sigma_{f}\right)\|B\|\left\|P_{(x(r), z(r), y(r))}(r)\right\|}{\sigma \sigma_{f} \sigma_{g} c(r)}\right)|c(t)-c(r)| .
\end{aligned}
$$

Therefore, there exists $L_{5}:=L_{5}(T)>0$ such that $\|v(t, r)-v(r, r)\| \leq L_{5}|c(t)-c(r)|$.

Futher by using (B.4) and (B.7), we have

$$
\begin{aligned}
\| w & (t, r)-w(r, r) \| \\
= & \| \sigma(c(t)-c(r)) b-\sigma(c(t)-c(r)) A x(r) \\
& -\sigma(c(t)-c(r)) B z(r)-\sigma A(c(t) u(t, r)-c(r) u(r, r)) \\
& -\sigma B(c(t) v(t, r)-c(r) v(r, r)) \| \\
\leq & \sigma\|b\||c(t)-c(r)|+\sigma\|A\|\|x(r)\||c(t)-c(r)| \\
& +\sigma\|B\|\|z(r)\||c(t)-c(r)| \\
& +\sigma\|A(c(t) u(t, r)-c(r) u(r, r))\| \\
& +\sigma\|B(c(t) v(t, r)-c(r) v(r, r))\| \\
\leq & \sigma(\|b\|+\|A\|\|x(r)\| \\
& +\|B\|\|z(r)\|)|c(t)-c(r)| \\
& +\sigma\|A(c(t) u(t, r)-c(r) u(t, r))\| \\
& +\sigma\|A(c(r) u(t, r)-c(r) u(r, r))\| \\
& +\sigma\|B(c(t) v(t, r)-c(r) v(t, r))\| \\
& +\sigma\|B(c(r) v(t, r)-c(r) v(r, r))\| \\
\leq & \sigma(\|b\|+\|A\|\|x(r)\|+\|B\|\|z(r)\|+\|A\|\|u(t, r)\| \\
& +\|A\|\|c(r)\| L_{4}+\|B\|\|v(t, r)\| \\
& \left.+\|B\|\|c(r)\| L_{5}\right)|c(t)-c(r)| .
\end{aligned}
$$

So there exists $L_{6}:=L_{6}(T)=\sup _{r \in[0, T]} \sigma(\|b\|+$ $\|A\|\|x(r)\|+\|B\|\|z(r)\|+\|A\|\|u(t, r)\|+\|A\|\|c(r)\| L_{4}$ $\left.+\|B\|\|v(t, r)\|+\|B\|\|c(r)\| L_{5}\right)>0$ such that

$\|w(t, r)-w(r, r)\| \leq L_{6}|c(t)-c(r)|$. 
Summing the relations (B.1)-(B.8) we get that there exists $L_{7}:=L_{7}(T)>0$ such that

$$
\begin{aligned}
& \|\dot{U}(t)-\dot{U}(r)\| \\
& \leq L_{7}(\|x(t)-x(r)\|+\|z(t)-z(r)\|+\|y(t)-y(r)\| \\
& \quad+|c(t)-c(r)|) .
\end{aligned}
$$

According to Remark 2(b), we can get $\dot{U}(\cdot)=(\dot{x}(\cdot), \dot{z}(\cdot), \dot{y}(\cdot))$ is absolutely continuous on $[0, T]$. This proves that the second order derivatives $(\ddot{x}(t), \ddot{z}(t), \ddot{y}(t))$ exists for almost every $t \in[0,+\infty)$.

In the next we prove the second statement. Note that $c(t)$ is bounded for all $t \in[0,+\infty)$. Then $L_{1}, L_{2}$ and $L_{3}$ can be taken as being global constants, so that (B.1),(B.2) and (B.6) hold for every $t, r \in[0,+\infty)$.

Since $R_{(x(r), z(r), y(r))}(r)=\dot{x}(r), Q_{(x(r), z(r), y(r))}(r)=\dot{z}(r)$ and $P_{(x(r), z(r), y(r))}(r)=\dot{y}(r)$ for every $r \in[0,+\infty)$. and taking into account (B.4) and (B.7) we get

$$
\begin{aligned}
& \|u(t, r)-u(r, r)\| \\
& \leq\left(\frac{\|A\|\|B\|\|\dot{z}(r)\|}{\sigma_{f}}+\frac{\|A\|\|\dot{y}(r)\|}{\sigma \sigma_{f} c(r)}\right)|c(t)-c(r)|,
\end{aligned}
$$

and, respectively,

$$
\begin{aligned}
& \|v(t, r)-v(r, r)\| \\
& \leq\left(\frac{c(t)\|A\|^{2}\|B\|^{2}\|\dot{z}(r)\|}{\sigma_{f} \sigma_{g}}\right. \\
& \left.\quad+\frac{\left(c(t)\|A\|^{2}+\sigma_{f}\right)\|B\|\|\dot{y}(r)\|}{\sigma \sigma_{f} \sigma_{g} c(r)}\right)|c(t)-c(r)|
\end{aligned}
$$

for every $t, r \in[0,+\infty)$. It holds

$$
\begin{aligned}
& \|\dot{x}(t)-\dot{x}(r)\|+\|\dot{z}(t)-\dot{z}(r)\| \\
& =\|u(t, t)-u(r, r)\|+\|v(t, t)-v(r, r)\| \\
& \leq\|u(t, t)-u(t, r)\|+\|u(t, r)-u(r, r)\| \\
& \quad+\|v(t, t)-v(t, r)\|+\|v(t, r)-v(r, r)\| .
\end{aligned}
$$

So, it follows from(B.1),(B.2),(B.9) and (B.10) that there exists $L>0$ such that

$$
\begin{aligned}
& \|\dot{x}(t)-\dot{x}(r)\|+\|\dot{z}(t)-\dot{z}(r)\| \\
& \leq L(\|x(t)-x(r)\|+\|z(t)-z(r)\|+\|y(t)-y(r)\| \\
& \quad+(\|\dot{z}(r)\|+\|\dot{y}(r)\|)|c(t)-c(r)|)
\end{aligned}
$$

for every $t, r \in[0,+\infty)$. Now we fix $r \in[0,+\infty)$ at which the second derivative of the trajectories exists and take in the above inequality $t=r+h$ for some $h>0$. Then

$$
\begin{aligned}
& \|\dot{x}(r+h)-\dot{x}(r)\|+\|\dot{z}(r+h)-\dot{z}(r)\| \\
& \leq L(\|x(r+h)-x(r)\|+\|z(r+h)-z(r)\|+ \\
& \|y(r+h)-y(r)\|+(\|\dot{z}(r)\|+\|\dot{y}(r)\|)|c(r+h)-c(r)|) .
\end{aligned}
$$

After dividing in the above inequality by $h$ and letting $h \rightarrow 0$ we obtain

$$
\begin{aligned}
& \|\ddot{x}(r)\|+\|\ddot{z}(r)\| \\
& \leq L(\|\dot{x}(r)\|+\|\dot{z}(r)\|+\|\dot{y}(r)\|+(\|\dot{z}(r)\|+\|\dot{y}(r)\|)|\dot{c}(r)|)
\end{aligned}
$$

and the proof is complete.

\section{Theorem 2}

Proof We need an appropriate energy functional in order to conclude. This will be accomplished in (B.18) below. Let $\left(x^{*}, z^{*}, y^{*}\right) \in \mathcal{H} \times \mathcal{G} \times \mathcal{K}$ be a saddle point of the Lagrangian $\mathcal{L}$. Then it fulfills the system of the optimality conditions

$$
\left\{\begin{array}{c}
A^{*} y^{*} \in \partial f\left(x^{*}\right) \\
B^{*} y^{*} \in \partial g\left(z^{*}\right) \\
A x^{*}+B z^{*}=b .
\end{array}\right.
$$

From (2.1) we have for almost every $t \in[0,+\infty)$

$$
\begin{aligned}
& A^{*} y(t)-c(t) A^{*} A(\dot{x}(t)+x(t))-c(t) A^{*} B z(t)+c(t) A^{*} b \\
& \in \partial f(\dot{x}(t)+x(t)),
\end{aligned}
$$

and by taking into account the strong monotonicity of $\partial f$ we have

$$
\begin{aligned}
& \left\langle A^{*}\left(y(t)-y^{*}\right)-c(t) A^{*} A(\dot{x}(t)+x(t))-c(t) A^{*} B z(t)\right. \\
& \left.+c(t) A^{*} b, \dot{x}(t)+x(t)-x^{*}\right\rangle \geq \sigma_{f}\left\|\dot{x}(t)+x(t)-x^{*}\right\|^{2} .
\end{aligned}
$$

In an analog way, according to (2.2) we have for almost every $t \in[0,+\infty)$

$$
\begin{aligned}
& B^{*} y(t)-c(t) B^{*} A(\dot{x}(t)+x(t))-c(t) B^{*} B(\dot{z}(t)+z(t)) \\
& +c(t) B^{*} b \in \partial g(\dot{z}(t)+z(t)),
\end{aligned}
$$

and by taking into account the strong monotonicity of $\partial g$ we have

$$
\begin{aligned}
& \left\langle B^{*}\left(y(t)-y^{*}\right)-c(t) B^{*} A(\dot{x}(t)+x(t))-c(t) B^{*} B(\dot{z}(t)\right. \\
& \left.+z(t))+c(t) B^{*} b, \dot{z}(t)+z(t)-z^{*}\right\rangle \\
& \geq \sigma_{g}\left\|\dot{z}(t)+z(t)-z^{*}\right\|^{2} .
\end{aligned}
$$

We use the last equation of (1.5) and the optimality condition $A x^{*}+B z^{*}=b$ to obtain for almost every $t \in[0,+\infty)$

$$
\begin{aligned}
&\left\langle A^{*}\left(y(t)-y^{*}\right), \dot{x}(t)+x(t)-x^{*}\right\rangle \\
&+\left\langle B^{*}\left(y(t)-y^{*}\right), \dot{z}(t)+z(t)-z^{*}\right\rangle \\
&=-\left\langle y(t)-y^{*},-A(\dot{x}(t)+x(t))+A x^{*}\right. \\
&\left.-B(\dot{z}(t)+z(t))+B z^{*}\right\rangle \\
&=-\frac{1}{\sigma c(t)}\left\langle y(t)-y^{*}, \dot{y}(t)\right\rangle \\
&=-\frac{1}{2 \sigma c(t)} \frac{d}{d t}\left\|y(t)-y^{*}\right\|^{2} .
\end{aligned}
$$


By summing up (B.11) and (B.12) and by taking into account (B.13), we obtain for almost every $t \in[0,+\infty)$

$$
\begin{aligned}
0 \leq & \left\langle-c(t) A^{*} A(\dot{x}(t)+x(t))-c(t) A^{*} B z(t)+c(t) A^{*} b,\right. \\
& \left.\dot{x}(t)+x(t)-x^{*}\right\rangle+\left\langle-c(t) B^{*} A(\dot{x}(t)+x(t))\right. \\
& \left.-c(t) B^{*} B(\dot{z}(t)+z(t))+c(t) B^{*} b, \dot{z}(t)+z(t)-z^{*}\right\rangle \\
& -\sigma_{f}\left\|\dot{x}(t)+x(t)-x^{*}\right\|^{2}-\sigma_{g}\left\|\dot{z}(t)+z(t)-z^{*}\right\|^{2} \\
& -\frac{1}{2 \sigma c(t)} \frac{d}{d t}\left\|y(t)-y^{*}\right\|^{2} .
\end{aligned}
$$

$$
\begin{aligned}
= & -\frac{1}{2 \sigma^{2} c(t)}\|\dot{y}(t)\|^{2}-\frac{c(t)}{2}\left\|A x(t)-A x^{*}\right\|^{2} \\
& -\frac{c(t)}{2}\|A \dot{x}(t)\|^{2}-\frac{c(t)}{2} \frac{d}{d t}\left\|A x(t)-A x^{*}\right\|^{2} \\
& +\left(\frac{c(t)\|B\|^{2}}{2}-\sigma_{g}\right)\left(\left\|z(t)-z^{*}\right\|^{2}+\|\dot{z}(t)\|^{2}\right. \\
& \left.+\frac{d}{d t}\left\|z(t)-z^{*}\right\|^{2}\right)+\left\langle c(t) A^{*} B \dot{z}(t), \dot{x}(t)+x(t)-x^{*}\right\rangle .
\end{aligned}
$$

We have for almost every $t \in[0,+\infty$ ) (Using the last equality for $\dot{y}$ in (1.5)):

$$
\begin{aligned}
& \left\langle-c(t) A^{*} A(\dot{x}(t)+x(t))-c(t) A^{*} B z(t)+c(t) A^{*} b, \dot{x}(t)\right. \\
& \left.+x(t)-x^{*}\right\rangle-\sigma_{g}\left\|\dot{z}(t)+z(t)-z^{*}\right\|^{2} \\
& =-\frac{1}{\sigma^{2} c(t)}\left\langle\dot{y}(t),-\sigma c(t) A\left(\dot{x}(t)+x(t)-x^{*}\right)\right\rangle \\
& +\left\langle c(t) A^{*} B \dot{z}(t), \dot{x}(t)+x(t)-x^{*}\right\rangle \\
& -\sigma_{g}\left\|\dot{z}(t)+z(t)-z^{*}\right\|^{2} \\
& =-\frac{1}{\sigma^{2} c(t)}\left[\frac{1}{2}\|\dot{y}(t)\|^{2}+\frac{1}{2}\left\|\sigma c(t) A\left(\dot{x}(t)+x(t)-x^{*}\right)\right\|^{2}\right. \\
& \left.-\frac{1}{2}\left\|\dot{y}(t)+\sigma c(t) A\left(\dot{x}(t)+x(t)-x^{*}\right)\right\|^{2}\right] \\
& +\left\langle c(t) A^{*} B \dot{z}(t), \dot{x}(t)+x(t)-x^{*}\right\rangle \\
& -\sigma_{g}\left\|\dot{z}(t)+z(t)-z^{*}\right\|^{2} \\
& =-\frac{1}{\sigma^{2} c(t)}\left[\frac{1}{2}\|\dot{y}(t)\|^{2}+\frac{1}{2} \sigma^{2} c^{2}(t)\left[\left\|A\left(x(t)-x^{*}\right)\right\|^{2}\right.\right. \\
& \left.+\|A \dot{x}(t)\|^{2}+2\left\langle\dot{x}(t), A^{*} A\left(x(t)-x^{*}\right)\right\rangle\right] \\
& \left.-\frac{1}{2}\left\|\sigma c(t)\left(b-A x^{*}-B(\dot{z}(t)+z(t))\right)\right\|^{2}\right] \\
& +\left\langle c(t) A^{*} B \dot{z}(t), \dot{x}(t)+x(t)-x^{*}\right\rangle \\
& -\sigma_{g}\left\|\dot{z}(t)+z(t)-z^{*}\right\|^{2} \\
& =-\frac{1}{\sigma^{2} c(t)}\left[\frac{1}{2}\|\dot{y}(t)\|^{2}+\frac{1}{2} \sigma^{2} c^{2}(t)\left[\left\|A\left(x(t)-x^{*}\right)\right\|^{2}\right.\right. \\
& \left.+\|A \dot{x}(t)\|^{2}+\frac{d}{d t}\left\|A x(t)-A x^{*}\right\|^{2}\right] \\
& \left.-\frac{1}{2}\left\|\sigma c(t)\left(B z^{*}-B(\dot{z}(t)+z(t))\right)\right\|^{2}\right] \\
& +\left\langle c(t) A^{*} B \dot{z}(t), \dot{x}(t)+x(t)-x^{*}\right\rangle \\
& -\sigma_{g}\left\|\dot{z}(t)+z(t)-z^{*}\right\|^{2} \\
& \leq-\frac{1}{\sigma^{2} c(t)}\left[\frac{1}{2}\|\dot{y}(t)\|^{2}+\frac{1}{2} \sigma^{2} c^{2}(t)\left[\left\|A x(t)-A x^{*}\right\|^{2}\right.\right. \\
& \left.\left.+\|A \dot{x}(t)\|^{2}+\frac{d}{d t}\left\|A x(t)-A x^{*}\right\|^{2}\right]\right] \\
& +\left\langle c(t) A^{*} B \dot{z}(t), \dot{x}(t)+x(t)-x^{*}\right\rangle \\
& +\left(\frac{c(t)\|B\|^{2}}{2}-\sigma_{g}\right)\left\|\dot{z}(t)+z(t)-z^{*}\right\|^{2}
\end{aligned}
$$

Similarly, for almost every $t \in[0,+\infty)$, by using the last equality for $\dot{y}$ in (1.5), we have

$$
\begin{aligned}
& \left\langle-c(t) B^{*} A(\dot{x}(t)+x(t))-c(t) B^{*} B(\dot{z}(t)+z(t))\right. \\
& \left.+c(t) B^{*} b, \dot{z}(t)+z(t)-z^{*}\right\rangle-\sigma_{f}\left\|\dot{x}(t)+x(t)-x^{*}\right\|^{2} \\
& =-\frac{1}{\sigma^{2} c(t)}\left\langle\dot{y}(t),-\sigma c(t) B\left(\dot{z}(t)+z(t)-z^{*}\right)\right\rangle \\
& -\sigma_{f}\left\|\dot{x}(t)+x(t)-x^{*}\right\|^{2} \\
& =-\frac{1}{\sigma^{2} c(t)}\left[\frac{1}{2}\|\dot{y}(t)\|^{2}+\frac{1}{2}\left\|\sigma c(t) B\left(\dot{z}(t)+z(t)-z^{*}\right)\right\|^{2}\right. \\
& \left.-\frac{1}{2}\left\|\dot{y}(t)+\sigma c(t) B\left(\dot{z}(t)+z(t)-z^{*}\right)\right\|^{2}\right] \\
& -\sigma_{f}\left\|\dot{x}(t)+x(t)-x^{*}\right\|^{2} \\
& =-\frac{1}{\sigma^{2} c(t)}\left[\frac{1}{2}\|\dot{y}(t)\|^{2}+\frac{1}{2} \sigma^{2} c^{2}(t)\left[\left\|B z(t)-B z^{*}\right\|^{2}\right.\right. \\
& \left.+\|B \dot{z}(t)\|^{2}+2\left\langle\dot{z}(t), B^{*} B\left(z(t)-z^{*}\right)\right\rangle\right] \\
& \left.-\frac{1}{2}\left\|\sigma c(t)\left(b-A(\dot{x}(t)+x(t))-B z^{*}\right)\right\|^{2}\right] \\
& -\sigma_{f}\left\|\dot{x}(t)+x(t)-x^{*}\right\|^{2} \\
& =-\frac{1}{\sigma^{2} c(t)}\left[\frac{1}{2}\|\dot{y}(t)\|^{2}+\frac{1}{2} \sigma^{2} c^{2}(t)\left[\left\|B z(t)-B z^{*}\right\|^{2}\right.\right. \\
& \left.+\|B \dot{z}(t)\|^{2}+2\left\langle B \dot{z}(t), B\left(z(t)-z^{*}\right)\right\rangle\right] \\
& \left.-\frac{1}{2}\left\|\sigma c(t)\left(A x^{*}-A(\dot{x}(t)+x(t))\right)\right\|^{2}\right] \\
& -\sigma_{f}\left\|\dot{x}(t)+x(t)-x^{*}\right\|^{2} \\
& \leq-\frac{1}{\sigma^{2} c(t)}\left[\frac{1}{2}\|\dot{y}(t)\|^{2}+\frac{1}{2} \sigma^{2} c^{2}(t)\left[\left\|B z(t)-B z^{*}\right\|^{2}\right.\right. \\
& \left.\left.+\|B \dot{z}(t)\|^{2}+2\left\langle B \dot{z}(t), B\left(z(t)-z^{*}\right)\right\rangle\right]\right] \\
& +\left(\frac{c(t)\|A\|^{2}}{2}-\sigma_{f}\right)\left\|\dot{x}(t)+x(t)-x^{*}\right\|^{2} \\
& =-\frac{1}{2 \sigma^{2} c(t)}\|\dot{y}(t)\|^{2}-\frac{c(t)}{2}\left\|B z(t)-B z^{*}\right\|^{2}-\frac{c(t)}{2}\|B \dot{z}(t)\|^{2} \\
& -\left\langle c(t) B \dot{z}(t), B\left(z(t)-z^{*}\right)\right\rangle+\left(\frac{c(t)\|A\|^{2}}{2}-\sigma_{f}\right)(\| x(t) \\
& \left.-x^{*}\left\|^{2}+\right\| \dot{x}(t)\left\|^{2}+\frac{d}{d t}\right\| x(t)-x^{*} \|^{2}\right) .
\end{aligned}
$$


By using the equation

$$
\begin{aligned}
\langle B \dot{z}(t), \dot{y}(t)\rangle= & \left\|\frac{\sqrt{m c(t)}}{2} B \dot{z}(t)+\frac{1}{\sqrt{m c(t)}} \dot{y}(t)\right\|^{2} \\
& -\frac{m c(t)}{4}\|B \dot{z}(t)\|^{2}-\frac{1}{m c(t)}\|\dot{y}(t)\|^{2}, m>0
\end{aligned}
$$

we have for almost every $t \in[0,+\infty)$ :

$$
\begin{aligned}
&\left\langle c(t) A^{*} B \dot{z}(t), \dot{x}(t)+x(t)-x^{*}\right\rangle-\left\langle c(t) B \dot{z}(t), B\left(z(t)-z^{*}\right)\right\rangle \\
&=\left\langle c(t) B \dot{z}(t), b-\frac{1}{\sigma c(t)} \dot{y}(t)-B(\dot{z}(t)+z(t))-A x^{*}\right\rangle \\
&-\left\langle c(t) B \dot{z}(t), B\left(z(t)-z^{*}\right)\right\rangle \\
&=\left\langle c(t) B \dot{z}(t), b-A x^{*}-B\left(z(t)-z^{*}\right)\right\rangle-\frac{1}{\sigma}\langle B \dot{z}(t), \dot{y}(t)\rangle \\
&-\langle c(t) B \dot{z}(t), B \dot{z}(t)\rangle-\langle c(t) B \dot{z}(t), B z(t)\rangle \\
&=-\left\langle c(t) B \dot{z}(t), B z(t)-2 B z^{*}\right\rangle-\frac{1}{\sigma}\left[\| \frac{\sqrt{m c(t)}}{2} B \dot{z}(t)\right. \\
&\left.+\frac{1}{\sqrt{m c(t)}} \dot{y}(t)\left\|^{2}-\frac{m c(t)}{4}\right\| B \dot{z}(t)\left\|^{2}-\frac{1}{m c(t)}\right\| \dot{y}(t) \|^{2}\right] \\
&-c(t)\|B \dot{z}(t)\|^{2}-\frac{c(t)}{2} \frac{d}{d t}\|B z(t)\|^{2} \\
&=-\frac{c(t)}{2} \frac{d}{d t}\left\|B z(t)-2 B z^{*}\right\|^{2}-\frac{1}{\sigma} \| \frac{\sqrt{m c(t)}}{2} B \dot{z}(t)+ \\
& \frac{1}{\sqrt{m c(t)}} \dot{y}(t)\left\|^{2}+\left(\frac{m}{4 \sigma}-1\right) c(t)\right\| B \dot{z}(t) \|^{2} \\
&+\frac{1}{m \sigma c(t)}\|\dot{y}(t)\|^{2}-\frac{c(t)}{2} \frac{d}{d t}\|B z(t)\|^{2} .
\end{aligned}
$$

By plugging the relations (B.15)-(B.17) into (B.14), we obtain for almost every $t \in[0,+\infty)$

$$
\begin{aligned}
0 \leq & -\frac{1}{2 \sigma^{2} c(t)}\|\dot{y}(t)\|^{2}-\frac{c(t)}{2}\left\|A x(t)-A x^{*}\right\|^{2} \\
& -\frac{c(t)}{2}\|A \dot{x}(t)\|^{2}-\frac{c(t)}{2} \frac{d}{d t}\left\|A x(t)-A x^{*}\right\|^{2} \\
& +\left(\frac{c(t)\|B\|^{2}}{2}-\sigma_{g}\right)\left(\left\|z(t)-z^{*}\right\|^{2}+\|\dot{z}(t)\|^{2}\right. \\
& \left.+\frac{d}{d t}\left\|z(t)-z^{*}\right\|^{2}\right)-\frac{1}{2 \sigma^{2} c(t)}\|\dot{y}(t)\|^{2} \\
& -\frac{c(t)}{2}\left\|B z(t)-B z^{*}\right\|^{2}-\frac{c(t)}{2}\|B \dot{z}(t)\|^{2} \\
& +\left(\frac{c(t)\|A\|^{2}}{2}-\sigma f\right)\left(\left\|x(t)-x^{*}\right\|^{2}+\|\dot{x}(t)\|^{2}\right. \\
& \left.+\frac{d}{d t}\left\|x(t)-x^{*}\right\|^{2}\right)-\frac{c(t)}{2} \frac{d}{d t}\left\|B z(t)-2 B z^{*}\right\|^{2} \\
& -\frac{1}{\sigma}\left\|\frac{\sqrt{m c(t)}}{2} B \dot{z}(t)+\frac{1}{\sqrt{m c(t)}} \dot{y}(t)\right\|^{2} \\
& +\left(\frac{m}{4 \sigma}-1\right) c(t)\|B \dot{z}(t)\|^{2}+\frac{1}{m \sigma c(t)}\|\dot{y}(t)\|^{2}
\end{aligned}
$$

$$
\begin{aligned}
& -\frac{c(t)}{2} \frac{d}{d t}\|B z(t)\|^{2}-\frac{1}{2 \sigma c(t)} \frac{d}{d t}\left\|y(t)-y^{*}\right\|^{2} \\
= & -\left(\frac{1}{\sigma^{2} c(t)}-\frac{1}{m \sigma c(t)}\right)\|\dot{y}(t)\|^{2}-\frac{c(t)}{2}\|A \dot{x}(t)\|^{2} \\
& -\left(\frac{3}{2}-\frac{m}{4 \sigma}\right) c(t)\|B \dot{z}(t)\|^{2}-\frac{c(t)}{2}\left\|A x(t)-A x^{*}\right\|^{2} \\
& -\frac{c(t)}{2}\left\|B z(t)-B z^{*}\right\|^{2} \\
& -\frac{1}{\sigma}\left\|\frac{\sqrt{m c(t)}}{2} B \dot{z}(t)+\frac{1}{\sqrt{m c(t)}} \dot{y}(t)\right\|^{2} \\
& +\left(\frac{c(t)\|A\|^{2}}{2}-\sigma_{f}\right)\left(\left\|x(t)-x^{*}\right\|^{2}+\|\dot{x}(t)\|^{2}\right) \\
& +\left(\frac{c(t)\|B\|^{2}}{2}-\sigma_{g}\right)\left(\left\|z(t)-z^{*}\right\|^{2}+\|\dot{z}(t)\|^{2}\right) \\
& -\frac{1}{2}\left(c(t) \frac{d}{d t}\left\|A x(t)-A x^{*}\right\|^{2}\right. \\
& +\left(2 \sigma_{f}-c(t)\|A\|^{2}\right) \frac{d}{d t}\left\|x(t)-x^{*}\right\|^{2} \\
& +c(t) \frac{d}{d t}\left\|B z(t)-2 B z^{*}\right\|^{2} \\
+ & \left(2 \sigma_{g}-c(t)\|B\|^{2}\right) \frac{d}{d t}\left\|z(t)-z^{*}\right\|^{2} \\
+ & \left.c(t) \frac{d}{d t}\|B z(t)\|^{2}+\frac{1}{\sigma c(t)} \frac{d}{d t}\left\|y(t)-y^{*}\right\|^{2}\right) .
\end{aligned}
$$

Taking into account that

$$
\begin{aligned}
& -\frac{1}{2}\left(c(t) \frac{d}{d t}\left\|A x(t)-A x^{*}\right\|^{2}\right. \\
& +\left(2 \sigma_{f}-c(t)\|A\|^{2}\right) \frac{d}{d t}\left\|x(t)-x^{*}\right\|^{2} \\
& +c(t) \frac{d}{d t}\left\|B z(t)-2 B z^{*}\right\|^{2} \\
& +\left(2 \sigma_{g}-c(t)\|B\|^{2}\right) \frac{d}{d t}\left\|z(t)-z^{*}\right\|^{2} \\
& \left.+c(t) \frac{d}{d t}\|B z(t)\|^{2}+\frac{1}{\sigma c(t)} \frac{d}{d t}\left\|y(t)-y^{*}\right\|^{2}\right) \\
& =-\frac{1}{2 \sigma c(t)}\left(\frac{d}{d t}\left(\sigma c^{2}(t)\left\|A x(t)-A x^{*}\right\|^{2}\right)\right. \\
& -2 \sigma c(t) \dot{c}(t)\left\|A x(t)-A x^{*}\right\|^{2} \\
& +\frac{d}{d t}\left(\sigma c(t)\left(2 \sigma f-c(t)\|A\|^{2}\right)\left\|x(t)-x^{*}\right\|^{2}\right) \\
& -2 \sigma \dot{c}(t)\left(\sigma \sigma_{f}-c(t)\|A\|^{2}\right)\left\|x(t)-x^{*}\right\|^{2} \\
& +\frac{d}{d t}\left(\sigma c^{2}(t)\left\|B z(t)-2 B z^{*}\right\|^{2}\right) \\
& -2 \sigma c(t) \dot{c}(t)\left\|B z(t)-2 B z^{*}\right\|^{2} \\
& +\frac{d}{d t}\left(\sigma c(t)\left(2 \sigma_{g}-c(t)\|B\|^{2}\right)\left\|z(t)-z^{*}\right\|^{2}\right) \\
& -2 \sigma \dot{c}(t)\left(\sigma_{g}-c(t)\|B\|^{2}\right)\left\|z(t)-z^{*}\right\|^{2} \\
& \left.+\sigma c^{2}(t) \frac{d}{d t}\|B z(t)\|^{2}+\frac{d}{d t}\left\|y(t)-y^{*}\right\|^{2}\right) \\
& \left.+{ }^{2}\right)
\end{aligned}
$$




$$
\begin{aligned}
= & -\frac{1}{2 \sigma c(t)} \frac{d}{d t}\left(\sigma c(t)\left(2 \sigma_{f}-c(t)\|A\|^{2}\right)\left\|x(t)-x^{*}\right\|^{2}\right. \\
& +\left\|x(t)-x^{*}\right\|_{\sigma c^{2}(t) A^{*} A}^{2}\left\|z(t)-2 z^{*}\right\|_{\sigma c^{2}(t) B^{*} B}^{2} \\
& +\sigma c(t)\left(2 \sigma_{g}-c(t)\|B\|^{2}\right)\left\|z(t)-z^{*}\right\|^{2} \\
& \left.+\sigma c^{2}(t)\|B z(t)\|^{2}+\left\|y(t)-y^{*}\right\|^{2}\right) \\
& +\dot{c}(t)\left(\left(\frac{\sigma_{f}}{c(t)}-\|A\|^{2}\right)\left\|x(t)-x^{*}\right\|^{2}+\left\|A x(t)-A x^{*}\right\|^{2}\right. \\
& \left.+\left(\frac{\sigma_{g}}{c(t)}-\|B\|^{2}\right)\left\|z(t)-z^{*}\right\|^{2}+\left\|B z(t)-2 B z^{*}\right\|^{2}\right),
\end{aligned}
$$

we obtain that

$$
\begin{aligned}
& 0 \leq-\frac{1}{2 \sigma c(t)} \frac{d}{d t}\left(\sigma c(t)\left(2 \sigma_{f}-c(t)\|A\|^{2}\right)\left\|x(t)-x^{*}\right\|^{2}\right. \\
& +\left\|x(t)-x^{*}\right\|_{\sigma c^{2}(t) A^{*} A}^{2}+\left\|z(t)-2 z^{*}\right\|_{\sigma c^{2}(t) B^{*} B}^{2} \\
& +\sigma c(t)\left(2 \sigma_{g}-c(t)\|B\|^{2}\right)\left\|z(t)-z^{*}\right\|^{2} \\
& \left.+\sigma c^{2}(t)\|B z(t)\|^{2}+\left\|y(t)-y^{*}\right\|^{2}\right) \\
& +\dot{c}(t)\left(\left(\frac{\sigma_{f}}{c(t)}-\|A\|^{2}\right)\left\|x(t)-x^{*}\right\|^{2}+\left\|A x(t)-A x^{*}\right\|^{2}\right. \\
& \left.+\left(\frac{\sigma_{g}}{c(t)}-\|B\|^{2}\right)\left\|z(t)-z^{*}\right\|^{2}+\left\|B z(t)-2 B z^{*}\right\|^{2}\right) \\
& -\left(\frac{1}{\sigma^{2} c(t)}-\frac{1}{m \sigma c(t)}\right)\|\dot{y}(t)\|^{2}-\frac{c(t)}{2}\|A \dot{x}(t)\|^{2} \\
& -\left(\frac{3}{2}-\frac{m}{4 \sigma}\right) c(t)\|B \dot{z}(t)\|^{2}-\frac{c(t)}{2}\left\|A x(t)-A x^{*}\right\|^{2} \\
& -\frac{c(t)}{2}\left\|B z(t)-B z^{*}\right\|^{2} \\
& -\frac{1}{\sigma}\left\|\frac{\sqrt{m c(t)}}{2} B \dot{z}(t)+\frac{1}{\sqrt{m c(t)}} \dot{y}(t)\right\|^{2} \\
& +\left(\frac{c(t)\|A\|^{2}}{2}-\sigma_{f}\right)\left(\left\|x(t)-x^{*}\right\|^{2}+\|\dot{x}(t)\|^{2}\right) \\
& +\left(\frac{c(t)\|B\|^{2}}{2}-\sigma_{g}\right)\left(\left\|z(t)-z^{*}\right\|^{2}+\|\dot{z}(t)\|^{2}\right) .
\end{aligned}
$$

Since $\dot{c}(t) \leq 0,0<c(t)<\min \left\{\frac{\sigma_{f}}{\|A\|^{2}}, \frac{\sigma_{g}}{\|B\|^{2}}\right\}$, if $c(t)$ is not constant (if $c(t)$ is constant we have $\dot{c}(t)=0$ ), let $\sigma<m<6 \sigma$, we have for almost every $t \in[0,+\infty)$.

$$
\begin{aligned}
0 \geq & \frac{1}{2} \frac{d}{d t}\left(\sigma c(t)\left(2 \sigma_{f}-c(t)\|A\|^{2}\right)\left\|x(t)-x^{*}\right\|^{2}\right. \\
& +\left\|x(t)-x^{*}\right\|_{\sigma c^{2}(t) A^{*} A}^{2}\left\|z(t)-2 z^{*}\right\|_{\sigma c^{2}(t) B^{*} B}^{2} \\
& +\sigma c(t)\left(2 \sigma_{g}-c(t)\|B\|^{2}\right)\left\|z(t)-z^{*}\right\|^{2} \\
& \left.+\sigma c^{2}(t)\|B z(t)\|^{2}+\left\|y(t)-y^{*}\right\|^{2}\right) \\
& +\left(\frac{1}{\sigma}-\frac{1}{m}\right)\|\dot{y}(t)\|^{2}+\frac{\sigma c^{2}(t)}{2}\|A \dot{x}(t)\|^{2} \\
& +\left(\frac{3 \sigma}{2}-\frac{m}{4}\right) c^{2}(t)\|B \dot{z}(t)\|^{2}+\frac{\sigma c^{2}(t)}{2}\left\|A x(t)-A x^{*}\right\|^{2} \\
& +\frac{\sigma c^{2}(t)}{2}\left\|B z(t)-B z^{*}\right\|^{2}
\end{aligned}
$$

$$
\begin{aligned}
& +c(t)\left\|\frac{\sqrt{m c(t)}}{2} B \dot{z}(t)+\frac{1}{\sqrt{m c(t)}} \dot{y}(t)\right\|^{2} \\
& +\sigma c(t)\left(\sigma_{f}-\frac{\|A\|^{2}}{2} c(t)\right)\left(\left\|x(t)-x^{*}\right\|^{2}+\|\dot{x}(t)\|^{2}\right) \\
& +\sigma c(t)\left(\sigma_{g}-\frac{\|B\|^{2}}{2} c(t)\right)\left(\left\|z(t)-z^{*}\right\|^{2}+\|\dot{z}(t)\|^{2}\right) .
\end{aligned}
$$

For $\underline{c}:=\epsilon$ and $\bar{c}:=\min \left\{\frac{2 \sigma_{f}}{\|A\|^{2}}, \frac{2 \sigma_{g}}{\|B\|^{2}}\right\}-\epsilon$ we have

$$
\begin{aligned}
0 \geq & \frac{1}{2} \frac{d}{d t}\left(\sigma c(t)\left(2 \sigma_{f}-c(t)\|A\|^{2}\right)\left\|x(t)-x^{*}\right\|^{2}\right. \\
& +\left\|x(t)-x^{*}\right\|_{\sigma c^{2}(t) A^{*} A}^{2}+\left\|z(t)-2 z^{*}\right\|_{\sigma c^{2}(t) B^{*} B}^{2} \\
& +\sigma c(t)\left(2 \sigma_{g}-c(t)\|B\|^{2}\right)\left\|z(t)-z^{*}\right\|^{2}+\sigma c^{2}(t)\|B z(t)\|^{2} \\
& \left.+\left\|y(t)-y^{*}\right\|^{2}\right)+\left(\frac{3 \sigma}{2}-\frac{m}{4}\right) \underline{c}^{2}\|B \dot{z}(t)\|^{2} \\
& +\frac{\sigma \underline{c}^{2}}{2}\left\|A x(t)-A x^{*}\right\|^{2}+\frac{\sigma \underline{c}^{2}}{2}\left\|B z(t)-B z^{*}\right\|^{2} \\
& +\underline{c}\left\|\frac{\sqrt{m \underline{c}}}{2} B \dot{z}(t)+\frac{1}{\sqrt{m \bar{c}}} \dot{y}(t)\right\|^{2}+\left(\frac{1}{\sigma}-\frac{1}{m}\right)\|\dot{y}(t)\|^{2} \\
& +\frac{\sigma \underline{c}}{2}\|A \dot{x}(t)\|^{2} \\
& +\sigma \underline{c}\left(\sigma_{f}-\frac{\|A\|^{2}}{2} \bar{c}\right)\left(\left\|x(t)-x^{*}\right\|^{2}+\|\dot{x}(t)\|^{2}\right) \\
& +\sigma \underline{c}\left(\sigma_{g}-\frac{\|B\|^{2}}{2} \bar{c}\right)\left(\left\|z(t)-z^{*}\right\|^{2}+\|\dot{z}(t)\|^{2}\right) .
\end{aligned}
$$

From Lemma 4 we have

$$
\begin{aligned}
\exists \lim _{t \rightarrow+\infty} & \left(\sigma c(t)\left(2 \sigma_{f}-c(t)\|A\|^{2}\right)\left\|x(t)-x^{*}\right\|^{2}\right. \\
& +\left\|x(t)-x^{*}\right\|_{\sigma c^{2}(t) A^{*} A}^{2} \\
& +\sigma c(t)\left(2 \sigma_{g}-c(t)\|B\|^{2}\right)\left\|z(t)-z^{*}\right\|^{2} \\
& +\left\|z(t)-2 z^{*}\right\|_{\sigma c^{2}(t) B^{*} B}^{2} \\
& \left.+\sigma c^{2}(t)\|B z(t)\|^{2}+\left\|y(t)-y^{*}\right\|^{2}\right) \in \Re .
\end{aligned}
$$

Let $T>0$. By integrating (B.18) on the interval $[0, T]$ we obtain

$$
\begin{aligned}
& \frac{1}{2}\left(\sigma c(T)\left(2 \sigma_{f}-c(T)\|A\|^{2}\right)\left\|x(T)-x^{*}\right\|^{2}\right. \\
& \quad+\left\|x(T)-x^{*}\right\|_{\sigma c^{2}(T) A^{*} A}^{2} \\
& \quad+\sigma c(T)\left(2 \sigma_{g}-c(T)\|B\|^{2}\right)\left\|z(T)-z^{*}\right\|^{2} \\
& \quad+\left\|z(T)-2 z^{*}\right\|_{\sigma c^{2}(T) B^{*} B}^{2} \\
& \left.\quad+\sigma c^{2}(T)\|B z(T)\|^{2}+\left\|y(T)-y^{*}\right\|^{2}\right) \\
& \quad+\left(\frac{3 \sigma}{2}-\frac{m}{4}\right) \underline{c}^{2} \int_{0}^{T}\|B \dot{z}(t)\|^{2} d t \\
& \quad+\frac{\sigma \underline{c}^{2}}{2} \int_{0}^{T}\left\|A x(t)-A x^{*}\right\|^{2} d t
\end{aligned}
$$




$$
\begin{aligned}
& +\frac{\sigma \underline{c}^{2}}{2} \int_{0}^{T}\left\|B z(t)-B z^{*}\right\|^{2} d t \\
& +\underline{c} \int_{0}^{T}\left\|\frac{\sqrt{m \underline{c}}}{2} B \dot{z}(t)+\frac{1}{\sqrt{m \bar{c}}} \dot{y}(t)\right\|^{2} d t \\
& +\left(\frac{1}{\sigma}-\frac{1}{m}\right) \int_{0}^{T}\|\dot{y}(t)\|^{2} d t+\frac{\sigma \underline{c}^{2}}{2} \int_{0}^{T}\|A \dot{x}(t)\|^{2} d t \\
& +\sigma \underline{c}\left(\sigma_{f}-\frac{\|A\|^{2}}{2} \bar{c}\right) \int_{0}^{T}\left(\left\|x(t)-x^{*}\right\|^{2}+\|\dot{x}(t)\|^{2}\right) d t \\
& +\sigma \underline{c}\left(\sigma_{g}-\frac{\|B\|^{2}}{2} \bar{c}\right) \int_{0}^{T}\left(\left\|z(t)-z^{*}\right\|^{2}+\|\dot{z}(t)\|^{2}\right) d t \\
& \leq \frac{1}{2}\left(\sigma c(0)\left(2 \sigma_{f}-c(0)\|A\|^{2}\right)\left\|x(0)-x^{*}\right\|^{2}\right. \\
& +\left\|x(0)-x^{*}\right\|_{\sigma c^{2}(0) A^{*} A}^{2}+\left\|z(0)-2 z^{*}\right\|_{\sigma c^{2}(0) B^{*} B}^{2} \\
& +\sigma c(0)\left(2 \sigma_{g}-c(0)\|B\|^{2}\right)\left\|z(0)-z^{*}\right\|^{2} \\
& \left.+\sigma c^{2}(0)\|B z(0)\|^{2}+\left\|y(0)-y^{*}\right\|^{2}\right) .
\end{aligned}
$$

Letting $T$ converge to $+\infty$ we have

$\|\dot{x}(\cdot)\|_{A^{*} A}^{2} \in L^{1}([0,+\infty), \Re),\|\dot{x}(\cdot)\|^{2} \in L^{1}([0,+\infty), \Re)$, $\|\dot{z}(\cdot)\|_{B^{*} B}^{2} \in L^{1}([0,+\infty), \Re),\|\dot{z}(\cdot)\|^{2} \in L^{1}([0,+\infty), \Re)$, $\left\|x(\cdot)-x^{*}\right\|^{2} \in L^{1}([0,+\infty), \Re)$,

$\left\|A x(t)-A x^{*}\right\|^{2} \in L^{1}([0,+\infty), \Re)$,

$\left\|z(\cdot)-z^{*}\right\|^{2} \in L^{1}([0,+\infty), \Re)$,

$\left\|B z(t)-B z^{*}\right\|^{2} \in L^{1}([0,+\infty), \Re)$,

$\|\dot{y}(\cdot)\|^{2} \in L^{1}([0,+\infty), \Re)$,

$\left\|\frac{\sqrt{3 \underline{c}}}{2} B \dot{z}(\cdot)+\frac{1}{\sqrt{3 \bar{c}}} \dot{y}(\cdot)\right\|^{2} \in L^{1}([0,+\infty), \Re)$,

and, consequently,

$\dot{x}(\cdot) \in L^{2}([0,+\infty), \mathcal{H}), \dot{z}(\cdot) \in L^{2}([0,+\infty), \mathcal{G})$,

$\dot{y}(\cdot) \in L^{2}([0,+\infty), \mathcal{K})$.

According to Lemma 3, this yields

$$
\ddot{x}(\cdot) \in L^{2}([0,+\infty), \mathcal{H}), \ddot{z}(\cdot), \ddot{y}(\cdot) \in L^{2}([0,+\infty), \mathcal{G}) .
$$

Consequently, for almost every $t \in[0,+\infty)$ it holds

$$
\frac{d}{d t}\|\dot{x}(t)\|^{2}=2\langle\ddot{x}(t), \dot{x}(t)\rangle \leq\left(\|\ddot{x}(t)\|^{2}+\|\dot{x}(t)\|^{2}\right),
$$

and the right-hand side is a function in $L^{1}([0,+\infty), \Re)$. Hence, according to Lemma 5,

$$
\lim _{t \rightarrow+\infty} \dot{x}(t)=0
$$

Similarly, we obtain that

$$
\lim _{t \rightarrow+\infty} \dot{z}(t)=0, \lim _{t \rightarrow+\infty} \dot{y}(t)=0 .
$$

In the following, let us prove that each weak sequential cluster point of $(x(t), z(t), y(t)), t \in[0,+\infty)$ is a saddle point of $\mathcal{L}$ (notice that the trajectories are bounded). Let $\left(x^{*}, z^{*}, \bar{y}\right)$ be such a weak sequentially cluster point. This means that there exists a sequence $\left(s_{n}\right)_{n \geq 0}$ with $\left(s_{n}\right) \rightarrow+\infty$ such that $\left(x\left(s_{n}\right), z\left(s_{n}\right), y\left(s_{n}\right)\right)$ converges to $\left(x^{*}, z^{*}, \bar{y}\right)$ as $n \rightarrow+\infty$ in the weak topology of $\mathcal{H} \times \mathcal{G} \times \mathcal{K}$. (notice that the trajectory $x(t), z(t)$ converges to $x^{*}, z^{*}$ strongly)

From (1.5) we have

$$
\begin{aligned}
b & -A\left(\dot{x}\left(s_{n}\right)+x\left(s_{n}\right)\right)-B\left(\dot{z}\left(s_{n}\right)+z\left(s_{n}\right)\right) \\
= & \frac{1}{\sigma c\left(s_{n}\right)} \dot{y}\left(s_{n}\right) \rightarrow 0 \quad(n \rightarrow \infty),
\end{aligned}
$$

and so it follows that $A x^{*}+B z^{*}=b$.

From (2.1) we have $\forall t \in[0,+\infty)$

$$
\begin{aligned}
& A^{*} y\left(s_{n}\right)-c\left(s_{n}\right) A^{*} A\left(\dot{x}\left(s_{n}\right)+x\left(s_{n}\right)\right)-c\left(s_{n}\right) A^{*} B z\left(s_{n}\right) \\
& +c\left(s_{n}\right) A^{*} b \in \partial f\left(\dot{x}\left(s_{n}\right)+x\left(s_{n}\right)\right) .
\end{aligned}
$$

Since $\left(y\left(s_{n}\right)\right)_{n \geq 0}$ converges weakly to $\bar{y}, \lim _{t \rightarrow+\infty} \dot{y}(t)$ $=0, \lim _{t \rightarrow+\infty} \dot{x}(t)=0, \lim _{t \rightarrow+\infty} x(t)=x^{*}, \lim _{t \rightarrow+\infty} \dot{z}(t)$ $=0, \lim _{t \rightarrow+\infty} z(t)=z^{*}$, and $A x^{*}+B z^{*}=b$, it follows from Proposition 20.33 in [9]

$$
A^{*} \bar{y} \in \partial f\left(x^{*}\right) \text {. }
$$

From (2.2) we have $\forall t \in[0,+\infty)$

$$
\begin{aligned}
& B^{*} y\left(s_{n}\right)-c\left(s_{n}\right) B^{*} A\left(\dot{x}\left(s_{n}\right)+x\left(s_{n}\right)\right)-c\left(s_{n}\right) B^{*} B\left(\dot{z}\left(s_{n}\right)\right. \\
& \left.+z\left(s_{n}\right)\right)+c\left(s_{n}\right) B^{*} b \in \partial g\left(\dot{z}\left(s_{n}\right)+z\left(s_{n}\right)\right) .
\end{aligned}
$$

Since $\left(y\left(s_{n}\right)\right)_{n \geq 0}$ converges weakly to $\bar{y}, \lim _{t \rightarrow+\infty} \dot{y}(t)$ $=0, \lim _{t \rightarrow+\infty} \dot{x}(t)=0, \lim _{t \rightarrow+\infty} x(t)=x^{*}, \lim _{t \rightarrow+\infty} \dot{z}(t)$ $=0, \lim _{t \rightarrow+\infty} z(t)=z^{*}$, and $A x^{*}+B z^{*}=b$, it follows from Proposition 20.33 in [9]

$$
B^{*} \bar{y} \in \partial g\left(z^{*}\right)
$$

In conclusion, $\left(x^{*}, z^{*}, \bar{y}\right)$ is a saddle point of the Lagrangian $\mathcal{L}$.

Now, we prove that $(x(t), z(t), y(t)), t \in[0,+\infty)$ converges weakly. So we consider two sequential cluster points $\left(x^{*}, z^{*}, y_{1}\right)$ and $\left(x^{*}, z^{*}, y_{2}\right)$. Consequently, there exists $\left(k_{n}\right)_{n \geq 0}$ and $\left(l_{n}\right)_{n \geq 0}$ such that the subsequence $\left(x\left(k_{n}\right), z\left(k_{n}\right), y\left(k_{n}\right)\right)$ converges weakly to $\left(x^{*}, z^{*}, y_{1}\right)$ as $n \rightarrow$ $+\infty$ and $\left(x\left(l_{n}\right), z\left(l_{n}\right), y\left(l_{n}\right)\right)$ converges weakly to $\left(x^{*}, z^{*}, y_{2}\right)$ as $n \rightarrow+\infty$, respectively. Well, based on the previous proof, we know that $\left(x^{*}, z^{*}, y_{1}\right)$ and $\left(x^{*}, z^{*}, y_{2}\right)$ are both saddle points of the Lagrangian L. From (B.19), we get $\exists \lim _{t \rightarrow+\infty}\left(\left\|y(t)-y_{1}\right\|^{2}-\left\|y(t)-y_{2}\right\|^{2}\right):=T$.

In the above equation, taking the limits along the subsequences $\left(k_{n}\right)_{n \geq 0}$ and $\left(l_{n}\right)_{n \geq 0}$ it yields

$$
T=-\left\|y_{2}-y_{1}\right\|^{2}=\left\|y_{2}-y_{1}\right\|^{2}
$$


so that

$$
\left\|y_{2}-y_{1}\right\|^{2}=0
$$

It follows that $y_{1}=y_{2}$. In consequence, $(x(t), z(t), y(t))$ converges weakly to a saddle point of the Lagrangian $\mathcal{L}$.

\section{Theorem 3}

Proof Let $\bar{x} \in \mathcal{H}, \bar{z} \in \mathcal{G}, \bar{x}+\bar{z}=b$ be fixed. By using (2.1), that is

$$
y(t)-c(\dot{x}(t)+x(t))-c z(t)+c b \in \partial f(\dot{x}(t)+x(t)),
$$

it yields

$$
\begin{aligned}
& f(\dot{x}(t)+x(t))-f(\bar{x}) \leq\langle-y(t)+c(\dot{x}(t)+x(t))+c z(t) \\
& \left.-c A^{*} b, \bar{x}-(\dot{x}(t)+x(t))\right\rangle-\frac{\sigma_{f}}{2}\|\bar{x}-(\dot{x}(t)+x(t))\|^{2} .
\end{aligned}
$$

for almost every $t \in(0,+\infty)$. Similarly, by using (2.2), that is

$y(t)-c(\dot{x}(t)+x(t))-c(\dot{z}(t)+z(t))+c b \in \partial g(\dot{z}(t)+z(t))$, it yields

$$
\begin{aligned}
& g(\dot{z}(t)+z(t))-g(\bar{z}) \leq\langle-y(t)+c(\dot{x}(t)+x(t))+c(\dot{z}(t) \\
& +z(t))-c b, \bar{z}-(\dot{z}(t)+z(t))\rangle-\frac{\sigma_{g}}{2}\|\bar{z}-(\dot{z}(t)+z(t))\|^{2},
\end{aligned}
$$

for almost every $t \in(0,+\infty)$. We recall the following two identities from the proof of 2 (here were actually replace $x^{*}$ with $\bar{x}, z^{*}$ with $\bar{z}$ and $y^{*}$ by 0 )

$$
\begin{aligned}
& \langle y(t), \dot{x}(t)+x(t)-\bar{x}\rangle+\langle y(t), \dot{z}(t)+z(t)-\bar{z}\rangle \\
& =-\langle y(t),-(\dot{x}(t)+x(t))+\bar{x}-(\dot{z}(t)+z(t))+\bar{z}\rangle \\
& =-\frac{1}{\sigma c}\langle y(t), \dot{y}(t)\rangle \\
& =-\frac{1}{2 \sigma c} \frac{d}{d t}\|y(t)\|^{2},
\end{aligned}
$$

which corresponds to (B.13).

$$
\begin{aligned}
& \langle-c(\dot{x}(t)+x(t))-c z(t)+c b, \dot{x}(t)+x(t)-\bar{x}\rangle \\
& -\frac{\sigma_{g}}{2}\|\dot{z}(t)+z(t)-\bar{z}\|^{2}+ \\
& \left\langle-c(\dot{x}(t)+x(t))-c(\dot{z}(t)+z(t))+c b, \dot{z}(t)+z(t)-z^{*}\right\rangle \\
& -\frac{\sigma_{f}}{2}\|\dot{x}(t)+x(t)-\bar{x}\|^{2} \\
& \leq-\frac{1}{2 \sigma^{2} c}\|\dot{y}(t)\|^{2}-\frac{c}{2}\|x(t)-\bar{x}\|^{2}-\frac{c}{2}\|\dot{x}(t)\|^{2} \\
& \quad-\frac{c}{2} \frac{d}{d t}\|x(t)-\bar{x}\|^{2}- \\
& \quad \frac{1}{2}\left(\sigma_{g}-c\right)\left(\|z(t)-\bar{z}\|^{2}+\|\dot{z}(t)\|^{2}+\frac{d}{d t}\|z(t)-\bar{z}\|^{2}\right)
\end{aligned}
$$

$$
\begin{aligned}
& -\frac{1}{2 \sigma^{2} c}\|\dot{y}(t)\|^{2}-\frac{c}{2}\|z(t)-\bar{z}\|^{2}-\frac{c}{2}\|\dot{z}(t)\|^{2}- \\
& \frac{1}{2}\left(\sigma_{f}-c\right)\left(\|x(t)-\bar{x}\|^{2}+\|\dot{x}(t)\|^{2}+\frac{d}{d t}\|x(t)-\bar{x}\|^{2}\right) \\
& -\frac{c}{2} \frac{d}{d t}\left\|z(t)-2 z^{*}\right\|^{2}-\frac{1}{\sigma}\left\|\frac{\sqrt{3 c}}{2} \dot{z}(t)+\frac{1}{\sqrt{3 c}} \dot{y}(t)\right\|^{2} \\
& +\left(\frac{3}{4 \sigma}-1\right) c\|\dot{z}(t)\|^{2}+\frac{1}{3 \sigma c}\|\dot{y}(t)\|^{2}-\frac{c}{2} \frac{d}{d t}\|z(t)\|^{2} \\
= & \frac{1}{\sigma c}\left(\frac{1}{m}-\frac{1}{\sigma}\right)\|\dot{y}(t)\|^{2}-\frac{c}{2}\|\dot{x}(t)\|^{2}-\frac{c}{2}\left(3-\frac{m}{2 \sigma}\right)\|\dot{z}(t)\|^{2} \\
& -\frac{\sigma_{f}}{2}\|x(t)-\bar{x}\|^{2}-\frac{\sigma_{g}}{2}\|z(t)-\bar{z}\|^{2} \\
& -\frac{1}{2}\left(\sigma \sigma_{f}-c\right)\|\dot{x}(t)\|^{2}-\frac{1}{2}\left(\sigma_{g}-c\right)\|\dot{z}(t)\|^{2} \\
& -\frac{c}{2}\left(\frac{d}{d t}\|x(t)-\bar{x}\|^{2}+\frac{d}{d t}\|x(t)-\bar{x}\|^{2}+\frac{d}{d t}\|z(t)-\bar{z}\|^{2}\right. \\
& \left.+\frac{d}{d t}\|z(t)-2 \bar{z}\|^{2}+\frac{d}{d t}\|z(t)\|^{2}\right)-\frac{\sigma_{f}}{2} \frac{d}{d t}\|x(t)-\bar{x}\|^{2} \\
& -\frac{\sigma_{g}}{2} \frac{d}{d t}\|z(t)-\bar{z}\|^{2}-\frac{1}{\sigma}\left\|\frac{\sqrt{m c}}{2} \dot{z}(t)+\frac{1}{\sqrt{m c}} \dot{y}(t)\right\|^{2} .
\end{aligned}
$$

That's what we get when we add (B.15), (B.16),(B.17) . By adding the two identities, (B.22) and (B.23), we obtain for almost every $t \in(0,+\infty)$

$$
\begin{aligned}
& (f(\dot{x}(t)+x(t))+g(\dot{z}(t)+z(t)))-(f(\bar{x})+g(\bar{z})) \\
& \leq \frac{1}{\sigma c}\left(\frac{1}{m}-\frac{1}{\sigma}\right)\|\dot{y}(t)\|^{2}-\frac{\sigma_{f}}{2}\|x(t)-\bar{x}\|^{2}-\frac{\sigma_{g}}{2}\|z(t)-\bar{z}\|^{2} \\
& \quad-\frac{1}{2} \sigma_{f}\|\dot{x}(t)\|^{2}-\frac{1}{2}\left(\sigma_{g}+2 c-\frac{m c}{2 \sigma}\right)\|\dot{z}(t)\|^{2} \\
& \quad-\frac{1}{\sigma}\left\|\frac{\sqrt{3 c}}{2} \dot{z}(t)+\frac{1}{\sqrt{3 c}} \dot{y}(t)\right\|^{2}-\frac{c}{2}\left(2 \frac{d}{d t}\|x(t)-\bar{x}\|^{2}\right. \\
& \left.\quad+\frac{d}{d t}\|z(t)-\bar{z}\|^{2}+\frac{d}{d t}\|2 z(t)-2 \bar{z}\|^{2}\right)- \\
& \quad \frac{\sigma_{f}}{2} \frac{d}{d t}\|x(t)-\bar{x}\|^{2}-\frac{\sigma_{g}}{2} \frac{d}{d t}\|z(t)-\bar{z}\|^{2}-\frac{1}{2 \sigma c} \frac{d}{d t}\|y(t)\|^{2} .
\end{aligned}
$$

By neglecting the negative terms, we obtain for almost every $t \in(0,+\infty)$

$$
\begin{aligned}
& (f(\dot{x}(t)+x(t))+g(\dot{z}(t)+z(t)))-(f(\bar{x})+g(\bar{z})) \\
& \leq-\frac{1}{2} \frac{d}{d t}\left(\|x(t)-\bar{x}\|_{\left(2 c+\sigma_{f}\right) I d}^{2}+\|z(t)-\bar{z}\|_{\left(3 c+\sigma_{g}\right) I d}^{2}\right. \\
& \left.\quad+\|y(t)\|_{\frac{1}{\sigma c} I d}^{2}\right),
\end{aligned}
$$

where

$$
W(t)=\left(\left(2 c+\sigma_{f}\right) I d,\left(3 c+\sigma_{g}\right) I d, \frac{1}{\sigma c} I d\right) .
$$

For $\tilde{x}(t)=\frac{1}{t} \int_{0}^{t}(\dot{x}(s)+x(s)) d s$ and $\tilde{z}(t)=\frac{1}{t} \int_{0}^{t}(\dot{z}(s)+$ $z(s)) d s$, it holds $\forall t \in(0,+\infty)$

$b-\tilde{x}-\tilde{z}=b-\frac{1}{t} \int_{0}^{t}((\dot{x}(s)+x(s))+(\dot{z}(s)+z(s))) d s$ 


$$
\begin{aligned}
& =b-\frac{1}{t} \int_{0}^{t}\left(b-\frac{1}{\sigma c} \dot{y}(s)\right) d s \\
& =\frac{1}{\sigma c t} \int_{0}^{t} \dot{y}(s) d s \\
& =\frac{y(t)-y_{0}}{\sigma c t} .
\end{aligned}
$$

From Theorem 2 it follows that the trajectory $(x(t), z(t)$, $y(t))$ converges weakly to a saddle point of $\mathcal{L}$ as $t \rightarrow$ $+\infty$. This means that $y(t), t \in[0,+\infty)$, it is bounded, thus there exists $K \geq 0$ such that

$$
\|b-\tilde{x}-\tilde{z}\| \leq \frac{K}{t}, \forall t \in(0,+\infty) .
$$

Let $t \in(0,+\infty)$ be such that $(\tilde{x}(t), \tilde{z}(t)) \in \operatorname{dom} f \times$ domg. By Jensen's inequality in the integral form we have for every $t \in(0,+\infty)$

$$
\begin{aligned}
f(\tilde{x}(t)) & =f\left(\frac{1}{t} \int_{0}^{t}(\dot{x}(s)+x(s)) d s\right) \\
& \leq \frac{1}{t} \int_{0}^{t} f(\dot{x}(s)+x(s)) d s
\end{aligned}
$$

and

$$
\begin{aligned}
g(\tilde{z}(t)) & =g\left(\frac{1}{t} \int_{0}^{t}(\dot{z}(s)+z(s)) d s\right) \\
& \leq \frac{1}{t} \int_{0}^{t} g(\dot{z}(s)+z(s)) d s,
\end{aligned}
$$

which, combined with (B.24), yields

$$
\begin{aligned}
f(\tilde{x}(t))+g(\tilde{z}(t)) & \\
\leq & \frac{1}{t} \int_{0}^{t}(f(\dot{x}(s)+x(s)) d s+g(\dot{z}(s)+z(s))) d s \\
\leq & \frac{1}{t} \int_{0}^{t}\left((f(\bar{x})+g(\bar{z}))-\frac{1}{2} \frac{d}{d s} \|(x(s), z(s), y(s))\right. \\
& \left.-(\bar{x}, \bar{z}, 0) \|_{W(s)}^{2}\right) d s \\
= & f(\bar{x})+g(\bar{z})-\frac{1}{2 t}\left(\|(x(t), z(t), y(t))-(\bar{x}, \bar{z}, 0)\|_{W(t)}^{2}\right. \\
& \left.-\|(x(0), z(0), y(0))-(\bar{x}, \bar{z}, 0)\|_{W(0)}^{2}\right) \\
\leq & f(\bar{x})+g(\bar{z})+\frac{\left\|\left(x^{0}, z^{0}, y^{0}\right)-(\bar{x}, \bar{z}, 0)\right\|_{W(0)}^{2}}{2 t} .
\end{aligned}
$$

Hence,

$$
\begin{aligned}
& (f(\tilde{x}(t))+g(\tilde{z}(t)))-(f(\bar{x})+g(\bar{z})) \\
& \leq \frac{\left\|\left(x^{0}, z^{0}, y^{0}\right)-(\bar{x}, \bar{z}, 0)\right\|_{W(0)}^{2}}{2 t} .
\end{aligned}
$$

Acknowledgements This work is supported by National Natural Science Foundation of China (Nos. 12061013,
71861002, 11601095), Natural Science Foundation of Guangxi Province (2016GXNSFBA380185), Training Plan of Thousands of Young and Middle-aged Backbone Teachers in Colleges and Universities of Guangxi, and Special Foundation for Guangxi Ba Gui Scholars.

Data availability statement Data sharing not applicable to this article as no datasets were generated or analysed during the current study.

\section{Ethics declarations}

Conflict of interest The authors of this paper confirm that any part of this work was not published or submitted for publication elsewhere, and authors do not have any conflict of interest with anybody else.

\section{References}

1. Peypouquet, J., Sorin, S.: Evolution equations for maximal monotone operators: asymptotic analysis in continuous and discrete time. J. Convex. Anal. 17(3-4), 1113-1163(2010)

2. Bot, R.I., Csetnek, E.R., László, S.C.: A primaldual dynamical approach to structured convex minimization problems. J. Differ. Equ. 269(12), 1071710757(2020)

3. Bitterlich, S., Csetnek, E.R., Wanka, G.: A dynamical approach to two-block separable convex optimization problems with linear constraints. Numer. Funct. Anal. Optim. 42, 1-38(2021)

4. Attouch, H.: Fast inertial proximal ADMM algorithms for convex structured optimization with linear constraint. https://hal.archivesouvertes.fr/hal-02501604(2020).

5. Abbas, B., Attouch,H.: Dynamical systems and forward-backward algorithms associated with the sum of a convex subdifferential and a monotone cocoercive operator. Optimization. 64(10), 22232252(2015)

6. Glowinski, R., Oden, J.T.: Numerical methods for nonlinear variational problems. Springer Berlin Heidelberg, German(1984)

7. Banert, S., Bot, R.I.: A forward-backward-forward differential equation and its asymptotic properties. J. Convex. Anal. 25(2), 371-388(2018)

8. Baillon, J.B., Brézis, H.: Une remarque sur le comportement asymptotique des semigroupes non linéaires. Houston. J. Math. 2(1), 5-7(1976)

9. Bauschke, H.H., Combettes, P.L.: Convex Analysis and Monotone Operator Theory in Hilbert Spaces. Springer International Publishing, New York(2017) 
10. Zalinescu, C.: Convex Analysis in General Vector Spaces. World Scientific Publishing Company, Singapore(2002)

11. Bot, R.I., Conjugate Duality in Convex Optimization. Springer, Berlin Heidelberg(2010)

12. Bauschke, H.H., Combettes, P.L.: Convex Analysis and Monotone Operator Theory in Hilbert Spaces. Second Edition. Springer, New York(2011)

13. Bot, R.I., Csetnek, E.R.: A dynamical system associated with the fixed points set of a nonexpansive operator. J. Dyn. Differ. Equ. 29(1), 155-168(2017)

14. Brézis, H.: Opérateurs maximaux monotones et semi-groupes de contractions dans les espaces de Hilbert. North-Holland/Elsevier, New York(1973)

15. Bruck, R.E.: Asymptotic convergence of nonlinear contraction semigroups in Hilbertspaces. J. Funct. Anal. 18, 15-26(1975)

16. Crandall, M.G., Pazy, A.: Semi-groups of nonlinear contractions and dissipativesets. J. Funct. Anal. 3, 376-418(1969)

17. Csetnek, E.R., Malitsky, Y., Tam, M.K.: Shadow Douglas-Rachford splitting for monotone inclusions. Appl. Math. Opt. 80, 665-678(2019)

18. Csetnek, E.R.: Continuous Dynamics Related to Monotone Inclusions and Non-Smooth Optimization Problems. Set-Valued. Var. Analy. 28, 32(2020)

19. Bot, R.I., Csetnek, E.R.: ADMM for monotone operators: convergence analysis and rates. Adv. Comput. Math. 45(1), 327-359(2019)

20. Attouch, H., Bot, R.I., Csetnek, E.R.: Fast optimization via inertial dynamics with closed-loop damping. arXiv preprint arXiv:2008.02261(2020)

21. Abbas, B., Attouch, H., Svaiter, B.F.: Newton-like dynamics and forward-backward methods for structured monotone inclusions in Hilbert spaces. J. Optimiz. Theory. App. 161(2), 331-360(2014)

22. Chao ,M.T., Han, D.R., Cai, X.J.: Convergence of the Peaceman-Rachford Splitting Method for a Class of Nonconvex Programs. Numer. Maththeory. Me. 14(2), 438-460(2021)

23. He, X., Hu, R., Fang, Y.P.: Fast convergence of primal-dual dynamics and algorithms with time scaling for linear equality constrained convex optimization problems. arXiv preprint arXiv:2103.12931(2021)

24. He, X.,Hu, R., Fang, Y.P.: Convergence rate analysis of fast primal-dual methods with scalings for linearly constrained convex optimization problems. arXiv preprint arXiv:2103.10118(2021)

25. Zhu, M., Hu, R., Fang, Y.P.: A continuous dynamical splitting method for solving 'strongly+weakly' convex programming problems. Optimization. 69(6), 1335-1359(2020) 